\title{
The eta-inverted sphere over the rationals
}

\author{
GLEN MATTHEW WILSON
}

\begin{abstract}
We calculate the motivic stable homotopy groups of the two-complete sphere spectrum after inverting multiplication by the Hopf map $\eta$ over fields of cohomological dimension at most 2 with characteristic different from 2 (this includes the $p$-adic fields $\mathbb{Q}_{p}$ and the finite fields $\mathbb{F}_{q}$ of odd characteristic) and the field of rational numbers; the ring structure is also determined.
\end{abstract}

14F42; 18G15, 55T15, 55Q45

\section{Introduction}

Guillou and Isaksen laid the foundation for calculating $\pi_{* *}\left(\mathbb{1}_{2}^{\wedge}\right)\left[\eta^{-1}\right]$, the motivic stable homotopy groups of the two-complete sphere spectrum after inverting multiplication by $\eta$, over the complex numbers using the $h_{1}$-inverted motivic Adams spectral sequence in [?]. They conjectured a pattern of differentials in the $h_{1}$-inverted motivic Adams spectral sequence and identified the $E_{\infty}$ page of the spectral sequence assuming the conjecture. Shortly after Guillou and Isaksen's paper appeared, Andrews and Miller [?] proved Guillou and Isaksen's conjecture. All together, these results show $\pi_{* *}\left(\mathbb{1}_{2}^{\wedge}\right)\left[\eta^{-1}\right] \cong \mathbb{F}_{2}\left[\eta^{ \pm 1}, \mu, \varepsilon\right] /\left(\varepsilon^{2}\right)$ [?, Conjecture 1.3]. Guillou and Isaksen then analyzed the $h_{1}$-inverted motivic Adams spectral sequence over the real numbers and gave a complete calculation of the ring $\pi_{* *}\left(\mathbb{1}_{2}^{\wedge}\right)\left[\eta^{-1}\right]$ over the base field $\mathbb{R}$ [?].

The subject of this paper is the calculation of $\pi_{* *}\left(\mathbb{1}_{2}^{\wedge}\right)\left[\eta^{-1}\right]$ over the field of rational numbers $\mathbb{Q}$ and fields $F$ with $\operatorname{cd}_{2}(F) \leq 2$ and characteristic different from 2, such as the $p$-adic fields $\mathbb{Q}_{p}$ and finite fields $\mathbb{F}_{q}$ of odd characteristic. We write $\pi_{s, w}\left(\mathbb{1}_{2}^{\wedge}\right)(F)$ for the stable homotopy group $\mathcal{S H}(F)\left(\Sigma^{s, w} \mathbb{1}, \mathbb{1}_{2}^{\wedge}\right)$ and frequently abbreviate this to $\pi_{s, w}\left(\mathbb{1}_{2}^{\wedge}\right)$ if the base field $F$ is clear from context.

Write $\mathfrak{M}(F)$ for the motivic Adams spectral sequence at the prime 2 over the field $F$ at the motivic sphere spectrum. This spectral sequence has $E_{2}$ page given by $\mathfrak{M}(F)_{2}^{f, s, w}=\operatorname{Ext}_{\mathcal{A}_{* *}(F)}^{f, s+f, w}\left(H_{* *}(F), H_{* *}(F)\right)$ and conditionally converges

$$
\mathfrak{M}(F)^{f, s, w} \Rightarrow \pi_{s, w}\left(\mathbb{1}_{H}^{\wedge}\right)(F)
$$


where $\mathbb{1}_{H}^{\wedge}$ is the $H$-nilpotent completion of the sphere spectrum defined by Bousfield [?, $\S 5]$. $\mathrm{Hu}, \mathrm{Kriz}$, and Ormsby [?, Theorem 1] proved that $\mathbb{1}_{H}^{\wedge}$ is weakly equivalent to $\mathbb{1}_{2}^{\wedge}$ over a field $F$ of characteristic 0 with $\operatorname{cd}_{2}(F[\sqrt{-1}])<\infty$. Wilson and Østvær [?, Proposition 5.10] note that the same argument works over fields over positive characteristic under the assumption that $\operatorname{cd}_{2}(F[\sqrt{-1}])<\infty$.

Given the conditionally convergent spectral sequence $\mathfrak{M}(F) \Rightarrow \pi_{* *}\left(\mathbb{1}_{2}^{\wedge}\right)(F)$ and that $\eta \in \pi_{1,1}\left(\mathbb{1}_{2}^{\wedge}\right)(F)$ is detected by $h_{1} \in \mathfrak{M}(F)^{1,1,1}$, one can try to calculate $\pi_{* *}\left(\mathbb{1}_{2}^{\wedge}\right)(F)\left[\eta^{-1}\right]$ using the $h_{1}$-inverted spectral sequence, defined as the following colimit of spectral sequences.

$$
\mathfrak{M}(F)\left[h_{1}^{-1}\right]=\operatorname{colim}\left(\mathfrak{M}(F) \stackrel{h_{1} \cdot}{\longrightarrow} \mathfrak{M}(F) \stackrel{h_{1} \cdot}{\longrightarrow} \mathfrak{M}(F) \cdots\right)
$$

It is not obvious that $\mathfrak{M}(F)\left[h_{1}^{-1}\right]$ converges to $\pi_{* *}\left(\mathbb{1}_{2}^{\wedge}\right)(F)\left[\eta^{-1}\right]$. Guillou and Isaksen show that it does converge for the complex numbers $\mathbb{C}$ in $[?, \S 6]$ and the real numbers $\mathbb{R}$ in $[?, \S 5]$. We address convergence for more general fields in section ??.

The Milnor-Witt $t$-stem of $\mathbb{1}_{2}^{\wedge}$ over $F$ is the group $\hat{\Pi}_{t}(F)=\bigoplus_{k \in \mathbb{Z}} \pi_{k+t, k}\left(\mathbb{1}_{2}^{\wedge}\right)(F)$. Note that $\hat{\Pi}_{0}(F)$ is a ring and $\hat{\Pi}_{t}(F)$ is a $\hat{\Pi}_{0}(F)$-module. Our main results will be stated in terms of Milnor-Witt stems and the Witt group of quadratic forms $W(F)$. In many cases, the two-complete $\eta$-inverted Milnor-Witt 0 -stem can be can be described in terms of the Witt ring of quadratic forms of the field.

Proposition 1 If $F$ is a field for which the Witt group of quadratic forms $W(F)$ is finitely generated or $W(F)$ has bounded 2-torsion exponent $(F=\mathbb{Q}$, for example), then there is an isomorphism $\hat{\Pi}_{0}(F)\left[\eta^{-1}\right] \cong W(F)_{2}^{\wedge}\left[\eta^{ \pm 1}\right]$.

Proof Morel has shown there is an isomorphism $\pi_{n, n}(\mathbb{1}) \cong W(F)$ for $n \geq 1$ in [?]. For $n \geq 1$ the homotopy group $\pi_{n, n}\left(\mathbb{1}_{2}^{\wedge}\right)$ fits into the exact sequence

$$
0 \rightarrow \operatorname{Ext}\left(\mathbb{Z} / 2^{\infty}, W(F)\right) \rightarrow \pi_{n, n}\left(\mathbb{1}_{2}^{\wedge}\right) \rightarrow \operatorname{Hom}\left(\mathbb{Z} / 2^{\infty}, \pi_{n-1, n}(\mathbb{1})\right) \rightarrow 0
$$

by [?, Equation (2)]. But as $\pi_{n-1, n} \mathbb{1}=0$ by Morel's connectivity theorem [?], there is an isomorphism $\operatorname{Ext}\left(\mathbb{Z} / 2^{\infty}, W(F)\right) \rightarrow \pi_{n, n}\left(\mathbb{1}_{2}^{\wedge}\right)$.

If $W(F)$ is finitely generated, there is an isomorphism $\operatorname{Ext}\left(\mathbb{Z} / 2^{\infty}, W(F)\right) \cong W(F)_{2}^{\wedge}$ by a result of Bousfield and Kan [?, Chapter VI, Section 2.1], hence $\pi_{n, n}\left(\mathbb{1}_{2}^{\wedge}\right) \cong W(F)_{2}^{\wedge}$. If $W(F)$ has bounded 2-torsion exponent, then $2^{n} W(F)=2^{m} W(F)$ for all $n$ and $m$ sufficiently large. The Mittag-Leffler condition is satisfied for the tower $\left\{2^{n} W(F)\right\}$, hence $\lim ^{1} 2^{n} W(F) \cong \lim ^{1} \operatorname{Hom}\left(\mathbb{Z} / 2^{n}, W(F)\right)$ vanishes. Now the short exact sequence of [?, Application 3.5.10]

$$
0 \rightarrow{\underset{\lim }{\longleftarrow}}^{1} \operatorname{Hom}\left(\mathbb{Z} / 2^{n}, W(F)\right) \rightarrow \operatorname{Ext}\left(\mathbb{Z} / 2^{\infty}, W(F)\right) \rightarrow W(F)_{2}^{\wedge} \rightarrow 0
$$


shows there is an isomorphism $\operatorname{Ext}\left(\mathbb{Z} / 2^{\infty}, W(F)\right) \cong W(F)_{2}^{\wedge}$, and so $\pi_{n, n}\left(\mathbb{1}_{2}^{\wedge}\right) \cong W(F)_{2}^{\wedge}$.

Finally, there is an isomorphism $\hat{\Pi}_{0}(F)\left[\eta^{-1}\right] \cong W(F)_{2}^{\wedge}\left[\eta^{ \pm 1}\right]$ since for any class $\alpha \in \hat{\Pi}_{0}(F)$ and $n$ sufficiently large, the class $\eta^{n} \alpha$ is an element of $\pi_{k, k} \mathbb{1} \cong W(F)$ with $k \geq 1$.

For finite fields $\mathbb{F}_{q}$, the Milnor-Witt 0 stem is now determined by the calculation of the Witt group of finite fields, a standard reference being Scharlau [?, Chapter 2, 3.3].

$$
\hat{\Pi}_{0}\left(\mathbb{F}_{q}\right)\left[\eta^{-1}\right] \cong W\left(\mathbb{F}_{q}\right)_{2}^{\wedge}\left[\eta^{ \pm 1}\right]= \begin{cases}\mathbb{Z} / 2\left[\eta^{ \pm 1}, u\right] / u^{2} & q \equiv 1 \bmod 4 \\ \mathbb{Z} / 4\left[\eta^{ \pm 1}\right] & q \equiv 3 \bmod 4\end{cases}
$$

We find in theorem ?? that for a field $F$ with $\operatorname{cd}_{2}(F) \leq 2$ and characteristic different from 2 the two-complete $\eta$-inverted Milnor-Witt stems take the following form.

$$
\hat{\Pi}_{t}(F)\left[\eta^{-1}\right] \cong \begin{cases}W(F)_{2}^{\wedge}\left[\eta^{ \pm 1}\right] & t \geq 0 \text { and } t \equiv 3 \bmod 4 \text { or } t \equiv 0 \bmod 4 \\ 0 & \text { otherwise }\end{cases}
$$

This gives a complete calculation of $\hat{\Pi}_{*}\left(\mathbb{F}_{q}\right)\left[\eta^{-1}\right]$ for the finite fields $\mathbb{F}_{q}$ of odd characteristic.

Theorem ?? calculates the ring $\hat{\Pi}_{*}(\mathbb{Q})\left[\eta^{-1}\right]$. In particular, the Milnor-Witt stems are

$$
\hat{\Pi}_{t}(\mathbb{Q})\left[\eta^{-1}\right] \cong \begin{cases}W(\mathbb{Q})_{2}^{\wedge}\left[\eta^{ \pm 1}\right] & t=0 \\ W(\mathbb{Q})_{2}^{\wedge}\left[\eta^{ \pm 1}\right] / 2^{n+1} & t \geq 0, t \equiv 3 \bmod 4, n=\nu_{2}(t+1) \\ M & t \equiv 0 \bmod 4, t \geq 4 \\ 0 & \text { otherwise }\end{cases}
$$

where $\nu_{2}(t+1)$ is the 2-adic valuation of $t+1$ and $M$ is the submodule of $W(\mathbb{Q})_{2}^{\wedge}\left[\eta^{ \pm 1}\right]$ defined in definition ??.

The method of proof for the calculations over $\mathbb{Q}$ of theorem ?? follows the strategy employed by Ormsby and Østvær in [?] to calculate the homotopy groups of $B P\langle n\rangle$ over $\mathbb{Q}$. First, for each completion $\mathbb{Q}_{\nu}$ of $\mathbb{Q}$ one uses the $\rho$-Bockstein spectral sequence to calculate $\operatorname{Ext}\left(\mathbb{Q}_{\nu}\right)\left[h_{1}^{-1}\right]$ and then the motivic Adams spectral sequence to calculate $\pi_{* *}\left(\mathbb{1}_{2}^{\wedge}\right)\left(\mathbb{Q}_{\nu}\right)\left[\eta^{-1}\right]$. We next follow the motivic Hasse principle to identify the differentials in the $\rho$-Bockstein spectral sequence and the motivic Adams spectral sequence over $\mathbb{Q}$ by comparing these spectral sequences with the associated spectral sequences over the completions.

The result of Ormsby, Röndigs, and Østvær, [?, Proof of Theorem 1.5] shows that the vanishing $\hat{\Pi}_{t}(\mathbb{Q})\left[\eta^{-1}\right]=0$ when $t \equiv 1,2 \bmod 4$ occurs systematically for all formally real fields $F$ with $\operatorname{cd}_{2}(F[i])<\infty$. Their calculation is used in this paper to show 
that $\hat{\Pi}_{1}(\mathbb{Q})\left[\eta^{-1}\right]$ vanishes, as it is unclear whether or not the motivic Adams spectral sequence over $\mathbb{Q}$ converges strongly in Milnor-Witt stem 1.

Ananyevskiy, Levine, and Panin investigate the $\eta$-inverted sphere spectrum $\mathbb{1}\left[\eta^{-1}\right]$ in [?] over fields $F$ of characteristic different from 2. They find that the stable homotopy sheaf $\oplus_{n \in \mathbb{Z}} \pi_{n, n} \mathbb{1}\left[\eta^{-1}\right]$ is isomorphic to the sheaf $\underline{W}\left[\eta^{ \pm 1}\right]$ where $\underline{W}$ is the Nisnevich sheaf associated to the presheaf of Witt groups (the Witt group $W(X)$ of an algebraic variety $X$ is defined by Knebusch in [?, Chapter I $\S 5])$. The consequence of this for calculating stable homotopy groups is that

$$
\oplus_{n \in \mathbb{Z}} \pi_{n, n}\left(\mathbb{1}\left[\eta^{-1}\right]\right)(F) \cong W(F)\left[\eta^{ \pm 1}\right]
$$

for all fields $F$ of characteristic different from 2. In addition to this absolute statement about the $\eta$-inverted Milnor-Witt 0 -stem, they identify the rationalization of $\mathbb{1}\left[\eta^{-1}\right]$ with an object in the heart of the homotopy $t$-structure on $\mathcal{S H}(F)$ [?, Theorem 3.4] and find that the sheaf $\pi_{s, w}\left(\mathbb{1}\left[\eta^{-1}\right]_{\mathbb{Q}}\right)$ takes the following form.

$$
\pi_{s, w}\left(\mathbb{1}\left[\eta^{-1}\right]_{\mathbb{Q}}\right)= \begin{cases}\underline{W}_{\mathbb{Q}} & \text { if } s=w \\ 0 & \text { otherwise }\end{cases}
$$

The calculations in this paper are about the $\eta$-inverted 2-complete sphere spectrum $\mathbb{1}_{2}^{\wedge}\left[\eta^{-1}\right]$ in contrast to Ananyevskiy, Levine, and Panin's results about $\mathbb{1}\left[\eta^{-1}\right]$ and $\mathbb{1}\left[\eta^{-1}\right]_{\mathbb{Q}}$.

We will follow the grading conventions for $\operatorname{Ext}(F)=\operatorname{Ext}_{\mathcal{A}_{* *}(F)}\left(H_{* *}(F), H_{* *}(F)\right)$ employed by Guillou and Isaksen in [?, §2.1]. In particular, for a class $x \in \operatorname{Ext}(F)$ in Adams filtration $f$, stem $s$, and weight $w$, the Milnor-Witt stem of $x$ is $t=s-w$ and the Chow weight of $x$ is $c=s+f-2 w$. We will write degrees as $\operatorname{deg}(x)=(f, t, c)$ unless otherwise specified. We will frequently use the isomorphism $\operatorname{Ext}(\mathbb{C})\left[h_{1}^{-1}\right] \cong$ $\mathbb{F}_{2}\left[h_{1}^{ \pm 1}, v_{1}^{4}, v_{2}, v_{3}, \ldots\right]$ with $\operatorname{deg}\left(v_{1}^{4}\right)=(4,4,4)$ and $\operatorname{deg}\left(v_{n}\right)=\left(1,2^{n}-1,1\right)$ established in [?, Theorem 3.4], and we adopt the convention of writing $P$ for $v_{1}^{4}$.

\section{Acknowledgments}

I would like to thank Dan Isaksen for teaching me how to use Massey product shuffles, Elden Elmanto for the motivation to write up the convergence result of section ??, Jonas Kylling and Håkon Kolderup for their comments on an earlier version of this work, Oliver Röndigs for helpful suggestions, and Paul Arne Østvær for his support and encouragement that made this paper possible. This paper was partially completed while participating in the research program "Algebro-geometric and homotopical methods" 
at the Institut Mittag-Leffler. I gratefully acknowledge support from the RCN project “Motivic Hopf Equations," no. 250399.

\section{Convergence of the $h_{1}$-inverted motivic Adams spectral sequence}

We refer the reader to Boardman's notes on spectral sequences [?] for the terminology concerning the convergence of spectral sequences. The notion of a map of filtered groups compatible with a map of spectral sequences is defined by Weibel in [?, p. 126].

Consider a collection of cohomologically graded spectral sequences $\left({ }^{i} \mathrm{E}_{r},{ }^{i} \mathrm{~d}_{r}\right)$ for $i \in \mathbb{N}$ where ${ }^{i} \mathrm{E}_{r}^{s}=0$ for all $s<0$ and all $r$, and each spectral sequence ${ }^{i} \mathrm{E}$ converges strongly to an abelian group ${ }^{i} \mathrm{G}$ filtered by ${ }^{i} \mathrm{~F}_{s}$. Here $r$ indicates the page of the spectral sequence and $s$ indicates the internal degree. We will omit these subscripts and superscripts where it is convenient. These assumptions on our spectral sequences precisely mean the following conditions hold.

(1) The filtration is exhaustive with ${ }^{i} \mathrm{~F}_{0}={ }^{i} \mathrm{G}$,

$$
{ }^{i} \mathrm{G}={ }^{i} \mathrm{~F}_{0} \supseteq{ }^{i} \mathrm{~F}_{1} \supseteq{ }^{i} \mathrm{~F}_{2} \supseteq \cdots
$$

(2) For all $s \in \mathbb{Z}$ there are isomorphisms ${ }^{i} \mathrm{E}_{\infty}^{s} \cong{ }^{i} \mathrm{~F}_{s} /{ }^{i} \mathrm{~F}_{s+1}$.

(3) The filtration is Hausdorff $\cap_{j}^{i} \mathrm{~F}_{j}=0$.

(4) The filtration is complete $\lim _{j}{ }_{j}^{i} \mathrm{G} /{ }^{i} \mathrm{~F}_{j} \cong{ }^{i} \mathrm{G}$.

We assume the spectral sequences are $\mathbb{Z}^{k}$-graded in addition to the internal grading $s$. In practice, $k$ is either 1 or 2 .

Let ${ }^{i} f:{ }^{i} \mathrm{E} \rightarrow{ }^{i+1} \mathrm{E}$ be a directed system of maps of spectral sequences compatible with ${ }^{i} g:{ }^{i} \mathrm{G} \rightarrow{ }^{i+1} \mathrm{G}$ of degree $a \in \mathbb{Z}^{k}$. The colimit of the directed system ${ }^{i} f:{ }^{i} \mathrm{E} \rightarrow{ }^{i+1} \mathrm{E}$ is again a spectral sequence $\widetilde{E}=\operatorname{colim}^{i}$ E that converges weakly to $\widetilde{G}=\operatorname{colim}^{i} \mathrm{G}$ filtered by $\widetilde{F}_{j}=\operatorname{colim}^{i} \mathrm{~F}_{j}$. Under what circumstances can we guarantee $\widetilde{E}$ converges strongly to $\widetilde{G}$ ?

The filtration $\widetilde{F}_{j}$ of $\widetilde{G}$ may fail to be Hausdorff or complete. To see how the Hausdorff condition may fail, consider ${ }^{i} \mathrm{E}=\mathbb{Z},{ }^{i} \mathrm{G}=\mathbb{Z}$, and ${ }^{i} \mathrm{~F}_{j}=0$ for $j \geq i$ and ${ }^{i} \mathrm{~F}_{j}={ }^{i} \mathrm{G}$ for $j<i$. If we define maps ${ }^{i} f=0$ and ${ }^{i} g(1)=1$, then $\widetilde{E}=0, \widetilde{G}=\mathbb{Z}$, and $\widetilde{F}_{j}=\mathbb{Z}$ for all $j \geq 0$. The issue is that the class $1 \in{ }^{i} \mathrm{G}$ is in filtration $i$, which shows $1 \in \widetilde{G}$ is in filtration $i$ for all natural numbers $i$. 
Completeness can fail if each ${ }^{i} \mathrm{G}$ has a finite filtration but the colimit $\widetilde{G}$ has an infinite filtration. Consider ${ }^{i} \mathrm{G}=\mathbb{Z},{ }^{i} \mathrm{~F}_{j}=2^{j} \mathbb{Z}$ for $j \leq i$ and ${ }^{i} \mathrm{~F}_{j}=0$ for $j>i$ with the map ${ }^{i} g(1)=1$. Then $\widetilde{G}=\mathbb{Z}$, yet $\widetilde{E}^{s} \cong \widetilde{F}_{s} / \widetilde{F}_{s+1} \cong \mathbb{Z} / 2$ for all $s \geq 0$ and $\lim _{s} \widetilde{G} / \widetilde{F}_{s}$ is isomorphic to the 2 -adic integers $\mathbb{Z}_{2}$.

Definition 2 Consider a directed system of $\mathbb{Z}^{k}$-graded spectral sequences ${ }^{i} f:{ }^{i} \mathrm{E} \rightarrow$ ${ }^{i+1} \mathrm{E}$ of degree $a$, that is, for all $i \in \mathbb{N}$ and $x \in{ }^{i} \mathrm{E}$ the degree of ${ }^{i} f(x)$ is $a+\operatorname{deg}(x)$, and ${ }^{i} f$ does not change the internal degree $s$. The directed system ${ }^{i} f$ has a horizontal vanishing line of height $N$ in the direction $a$ if for any degree $b$ there exists $K \in \mathbb{N}$ for which the groups ${ }^{i} \mathrm{E}^{s, b+i a}$ vanish for all $i>K$ and $s>N$.

The term horizontal vanishing line comes from the special case where for all $i$ we have ${ }^{i} E=E$ and $E=\oplus_{s, p} E^{s, p}$ is a $\mathbb{Z}$-graded spectral sequence in $p$ with internal degree $s$. If one makes a chart for ${ }^{i} E$ where the vertical axis is the internal degree $s$ and the horizontal axis is $p$, a horizontal vanishing line of height $N$ in the direction 1 says that $E^{s, p}$ vanishes when $(s, p)$ is above the horizontal line $s=N$ and $p$ is sufficiently large.

Proposition 3 Suppose ${ }^{i} f:{ }^{i} \mathrm{E} \rightarrow{ }^{i+1} \mathrm{E}$ is a directed system of $\mathbb{Z}^{k}$-graded spectral sequences of degree $a$ with a horizontal vanishing line of height $N$ in the direction $a$. The colimit spectral sequence $\widetilde{E}$ then converges strongly to $\widetilde{G}$ with respect to the filtration $\widetilde{F}$.

Proof We first show the filtration $\widetilde{F}$ of $\widetilde{G}$ is complete. For $b \in \mathbb{Z}^{k}$, the degree $b$ component of $\widetilde{G}$ is

$$
\widetilde{G}^{b}=\operatorname{colim}\left({ }^{0} G^{b} \rightarrow{ }^{1} G^{b+a} \rightarrow \cdots \rightarrow{ }^{i} \mathrm{G}^{b+i a} \rightarrow \cdots\right) .
$$

The assumption that there is a horizontal vanishing line of height $N$ in the direction $a$ implies for all $i>K$ the filtration of ${ }^{i} \mathrm{G}^{b+i a}$ is finite. This is because the filtration of ${ }^{i} \mathrm{G}$ is Hausdorff and ${ }^{i} E^{s, b+i a}$ vanishes for $s>N$, so ${ }^{i} \mathrm{~F}_{j}$ is trivial for $i>K$ and $j>N$. Since finite limits and directed colimits commute, it follows that

$$
\begin{aligned}
\lim _{j}\left(\widetilde{G}^{b} / \widetilde{F}_{j}^{b}\right) & \cong \lim _{j}\left(\operatorname{colim}_{i>K}{ }^{i} \mathrm{G} /{ }^{i} \mathrm{~F}_{j}\right) \\
& \cong \operatorname{colim}_{i>K}\left(\varlimsup_{j}{ }_{j} \mathrm{G} /{ }^{i} \mathrm{~F}_{j}\right) \\
& \cong \operatorname{colim}_{i>K}{ }^{i} \mathrm{G}^{b+i a} \\
& \cong \widetilde{G}^{b} .
\end{aligned}
$$

We now show the filtration $\widetilde{F}_{j}$ of $\widetilde{G}$ is Hausdorff. Let $x \in \widetilde{G}^{b}$ be a nonzero element. Lemma ?? shows there is some ${ }^{i} x \in{ }^{i} \mathrm{G}^{b+i a}$ which maps to $x \in \widetilde{G}^{b}$ for which ${ }^{i} x$ is 
detected by ${ }^{i} y \in{ }^{i} \mathrm{E}_{r}^{s, b+i a}$ and ${ }^{i+k+1} y$ is nonzero for all $k \in \mathbb{N}$. Since ${ }^{i} f$ is compatible with ${ }^{i} g$, it follows that ${ }^{i+k+1} y$ detects ${ }^{i+k+1} x={ }^{i+k} g \circ \cdots \circ{ }^{i} g\left({ }^{i} x\right)$ for all $k \in \mathbb{N}$. Furthermore, ${ }^{i+k} x \in{ }^{i+k} G$ is nonzero for all $k \in \mathbb{N}$ and so ${ }^{i+k} y$ survives to ${ }^{i+k} E_{\infty}^{s, b+(i+k) a}$. Our assumption that the spectral sequences ${ }^{i} \mathrm{E}$ converge strongly to ${ }^{i} \mathrm{G}$ means that

$$
{ }^{i+k} E_{\infty}^{s, b+(i+k) a} \cong{ }^{i+k} \mathrm{~F}_{s} /{ }^{i+k} \mathrm{~F}_{s+1} .
$$

Hence every class ${ }^{i+k} x$ is in filtration $s$ but not $s+1$, so that $x \in \widetilde{F}_{s}$ but $x \notin \widetilde{F}_{s+1}$. It now follows that the filtration $\widetilde{F}$ of $\widetilde{G}$ is Hausdorff.

Lemma 4 Under the conditions of proposition ??, consider a nonzero element $x \in \widetilde{G}^{b}$. There exists some ${ }^{i} x \in{ }^{i} \mathrm{G}^{b+i a}$ that maps to $x \in \widetilde{G}^{b}$ for which ${ }^{i} x$ is detected by ${ }^{i} y \in{ }^{i} \mathrm{E}_{r}^{s, b+i a}$ and

$$
{ }^{i+k+1} y={ }^{i+k} f \circ \cdots \circ{ }^{i} f\left({ }^{i} y\right) \in{ }^{i+k+1} E_{r}^{s, b+(i+k+1) a}
$$

is nonzero for all $k \in \mathbb{N}$.

Proof There is some ${ }^{j} x \in{ }^{j} \mathrm{G}$ which maps to $x \in \widetilde{G}$. The classes ${ }^{j+k} x={ }^{j+k-1} g \circ \cdots \circ$ ${ }^{j} g\left({ }^{j} x\right)$ are non-zero for all $k \geq 1$ and are therefore detected by some class ${ }^{j+k} y \in{ }^{j+k} \mathrm{E}^{s_{k}, *}$. The vanishing line implies $s_{k} \leq N$ for $k$ sufficiently large and the compatibility of the maps ${ }^{j} f$ with ${ }^{j} g$ implies $s_{k}$ is a non-decreasing function of $k$. Hence $s_{k}$ is eventually constant, say for all $k+j \geq i$. Then ${ }^{i} x$ has the desired property.

These results can be applied to inverting multiplication by $h_{1}$ in the motivic Adams spectral sequence at the prime 2 after re-indexing the filtration. For a field $F$, write ${ }^{i} \mathrm{E}^{f}$ for $\mathfrak{M}(F)^{f+i}$. Here $f$ is the internal degree of the spectral sequence ("f" for "filtration"). With this convention, the maps $h_{1} \cdot:{ }^{i} \mathrm{E} \rightarrow{ }^{i+1} \mathrm{E}$ form a directed system of spectral sequences which is compatible with the maps $\eta:{ }^{i} \mathrm{G} \rightarrow{ }^{i+1} \mathrm{G}$ where ${ }^{i} F_{j}=F_{j+i}\left(\pi_{* *}\left(\mathbb{1}_{2}^{\wedge}\right)\right)$. The degree of multiplication by $\eta$ is $(s, w)=(1,1)$ where $s$ is the stem and $w$ the weight. A horizontal vanishing line of height $N$ in the direction $(1,1)$ is equivalent to the following condition. For any $(s, w)$ there exists $k$ so that for all $i>k$ and $f>N$ the group $\mathfrak{M}(F)^{f+i, s+i, w+i}$ vanishes. In the usual manner of drawing a chart for $\mathfrak{M}(F)$, such as those made by Isaksen [?], the horizontal vanishing line for the system ${ }^{i} \mathrm{E}^{f}$ is transformed into a line of slope 1 .

Such vanishing conditions occur over $\mathbb{R}$ in positive Milnor-Witt stems as proved by Guillou and Isaksen [?, Lemma 5.1]. Over $\mathbb{R}$ it suffices to take $N=1$, but one must take larger values for other fields. For fields of cohomological dimension at most 2 and number fields, take $N=3$ for the positive Milnor-Witt stems. 
Corollary 5 The $h_{1}$-inverted motivic Adams spectral sequence over fields of cohomological dimension at most 2 and the field of rational numbers $\mathbb{Q}$ converges strongly to $\pi_{s, w}\left(\mathbb{1}_{2}^{\wedge}\right)\left[\eta^{-1}\right]$ when $s-w>1$.

Proof Consider the directed system ${ }^{i} \mathrm{E}^{f}=\mathfrak{M}(F)^{f+i}$ with maps $h_{1} \cdot:{ }^{i} \mathrm{E} \rightarrow{ }^{i+1} \mathrm{E}$ as described above. With $N=3$, the vanishing conditions required for proposition ?? are satisfied for fields $F$ of cohomological dimension at most 2. The $\rho$-Bockstein spectral sequence for such a field has $E_{1}$-page $H_{* *}(F) \otimes_{\mathbb{F}_{2}[\tau]} \operatorname{Ext}(\mathbb{C})$ and converges off to $\operatorname{Ext}(F)$. The $E_{1}$ page of the $\rho$-Bockstein spectral sequence has the claimed vanishing line in positive Milnor-Witt stem; hence $\operatorname{Ext}(F)$ does too.

An argument similar to the one given by Guillou and Isaksen in [?, Lemma 5.1] establishes a vanishing line over $\mathbb{Q}$ in positive Milnor-Witt stems with $N=3$. Their choice of $A$ works just as well over $\mathbb{Q}$ ( $A$ corresponds to $k$ when $s=0$ in the notation above) because the $\rho$-inverted Hopf algebroid $\left(H_{* *}(\mathbb{Q})\left[\rho^{-1}\right], \mathcal{A}_{* *}(\mathbb{Q})\left[\rho^{-1}\right]\right)$ is isomorphic to the $\rho$-inverted Hopf algebroid over $\mathbb{R}$. Their argument with the $\rho$-Bockstein spectral sequence must only be modified to account for $y$ being of the form $y=\alpha \tilde{y}$ with $\tilde{y} \in \operatorname{Ext}(\mathbb{C})$ and $\alpha \in H_{i, i}(\mathbb{Q})$ with $i \leq 2$ and $\alpha$ is not divisible by $\rho$.

The motivic Adams spectral sequence for fields $F$ with $\operatorname{cd}_{2}(F) \leq 2$ and $\mathbb{Q}$ converges conditionally to $\pi_{* *}\left(\mathbb{1}_{2}^{\wedge}\right)$ by [?, Theorem 1] of Hu, Kriz, and Ormsby. The vanishing line described above ensures that it also converges strongly in Milnor-Witt stem at least 2 , as in such degrees $d_{r}=0$ for $r$ sufficiently large. Hence we get the convergence result of the $h_{1}$-inverted Adams spectral sequences.

\section{Fields of cohomological dimension at most 2}

Let $F$ be a field of 2-cohomological dimension at most 2. The mod 2 Milnor $K$-theory of such a field satisfies $k_{n}^{M}(F)=0$ for $n \geq 3$. We first calculate $\operatorname{Ext}(F)\left[h_{1}^{-1}\right]$ using the $\rho$ Bockstein spectral sequence and then observe that the structure of $\mathfrak{M}(F)_{2} \cong \operatorname{Ext}(F)\left[h_{1}^{-1}\right]$ forces the $h_{1}$-inverted motivic Adams spectral sequence to collapse at the $E_{2}$ page. See figures ?? and ?? for a depiction of the $\rho$-Bockstein spectral sequence $E_{1}$ and $E_{\infty}$ page up to Milnor-Witt stem 24.

Proposition 6 For $F$ a field with $\operatorname{cd}_{2}(F) \leq 2$, the $E_{2}$ page of $\mathfrak{M}(F)\left[h_{1}^{-1}\right]$ is

$$
\operatorname{Ext}(F)\left[h_{1}^{-1}\right] \cong k_{*}^{M}(F) \otimes \operatorname{Ext}(\mathbb{C})\left[h_{1}^{-1}\right] .
$$


Proof If -1 is a square in $F$, it follows that $\operatorname{Ext}(F) \cong H_{* *}(F) \otimes_{\mathbb{F}_{2}[\tau]} \operatorname{Ext}(\mathbb{C})$ by an argument similar to [?, Proposition 7.1]. The class $\tau$ is killed after inverting $h_{1}$, so the result follows in this case.

If -1 is not a square in $F$, use the $\rho$-Bockstein spectral sequence. The $E_{1}$ page of the $h_{1}$-inverted $\rho$-Bockstein spectral sequence is

$$
E_{1}^{\epsilon, *, *, *} \cong \rho^{\epsilon} k_{*}^{M}(F) / \rho^{\epsilon+1} k_{*}^{M}(F) \otimes \operatorname{Ext}(\mathbb{C})\left[h_{1}^{-1}\right]
$$

and the $d_{r}$ differential has degree $(r, 1,-1,0)$ with respect to the grading $(\epsilon, f, t, c)$.

The differentials $d_{r}$ with $r \geq 1$ vanish on the generators $P=v_{1}^{4}$ and $v_{n}$ for $n \geq 2$ of $\operatorname{Ext}(\mathbb{C})\left[h_{1}^{-1}\right]$ for degree reasons. Any non-zero class $x \in \operatorname{Ext}(\mathbb{C})\left[h_{1}^{-1}\right]$ has $t+c \equiv$ $0 \bmod 4$, but the degree of $d_{r}(x)$ satisfies $t+c \equiv 3 \bmod 4$. If $F$ has cohomological dimension at most 2 , then any non-zero class in the $h_{1}$-inverted $\rho$-Bockstein spectral sequence satisfies $t+c \not \equiv 3 \bmod 4$. Hence the $h_{1}$-inverted $\rho$-Bockstein spectral sequence collapses. There is no possibility for hidden extensions, so the proposition follows.

Proposition 7 If $\bar{F}$ is an algebraically closed field of characteristic different from 2, the $\eta$-inverted motivic homotopy groups of spheres over $\bar{F}$ are given by

$$
\pi_{* *}\left(\mathbb{1}_{2}^{\wedge}\right)(\bar{F})\left[\eta^{-1}\right] \cong \pi_{* *}\left(\mathbb{1}_{2}^{\wedge}\right)(\mathbb{C})\left[\eta^{-1}\right] \cong \mathbb{F}_{2}\left[\eta^{ \pm 1}, \mu, \varepsilon\right] /\left(\varepsilon^{2}\right)
$$

where $\mu \in \pi_{9,5}\left(\mathbb{1}_{2}^{\wedge}\right)$ is the unique homotopy class detected by $P h_{1}$ and $\varepsilon \in \pi_{8,5}\left(\mathbb{1}_{2}^{\wedge}\right)$ is the unique homotopy class detected by $c_{0}$.

Proof If $\bar{F}$ is characteristic zero, there is an isomorphism $\mathfrak{M}(\bar{F}) \cong \mathfrak{M}(\mathbb{C})$ by the proof of [?, Lemma 6.4]. If $\bar{F}$ has positive characteristic, the change of characteristic argument [?, Corollary 6.1] comparing $\mathfrak{M}(\bar{F})$ to $\mathfrak{M}(\mathbb{C})$ via the motivic Adams spectral sequence over the ring of Witt vectors of $\bar{F}$ shows there is an isomorphism of spectral sequences $\mathfrak{M}(\bar{F}) \cong \mathfrak{M}(\mathbb{C})$. This isomorphism propagates to an isomorphism after inverting multiplication by $h_{1}$. The now resolved conjecture of Guillou and Isaksen in [?, Conjecture 1.3] gives the explicit description.

Proposition 8 The $d_{2}$ differentials for the $h_{1}$-inverted motivic Adams spectral sequence for a field $F$ with characteristic different from 2 and $\operatorname{cd}_{2}(F) \leq 2$ follow from $d_{2}\left(v_{n}\right)=$ $h_{1}^{2} v_{n-1}^{2}$ for $n \geq 3$ and $d_{2}(x)=0$ for $x \in k_{*}^{M}(F)$ by using the Leibniz rule. Furthermore, $\mathfrak{M}(F)\left[h_{1}^{-1}\right]$ collapses at the $E_{3}$ page.

Proof The inclusion of $F$ into its algebraic closure $\bar{F}$ induces a map of spectral sequences

$$
\Phi: \mathfrak{M}(F)\left[h_{1}^{-1}\right] \rightarrow \mathfrak{M}(\bar{F})\left[h_{1}^{-1}\right] \cong \mathfrak{M}(\mathbb{C})\left[h_{1}^{-1}\right]
$$


Andrews and Miller [?, Theorem 9.15] have proved that in $\mathfrak{M}(\mathbb{C})\left[h_{1}^{-1}\right]$ there are differentials $d_{2}\left(v_{n}\right)=h_{1}^{2} v_{n-1}^{2}$ for all $n \geq 3$. It follows that in $\mathfrak{M}(F)\left[h_{1}^{-1}\right]$ we must have $d_{2}\left(v_{n}\right)=h_{1}^{2} v_{n-1}^{2}$ up to some element in the kernel of the comparison map $\Phi$. A class $x \in \operatorname{ker}(\Phi)$ satisfies $t+c \equiv 1 \bmod 4$ or $t+c \equiv 2 \bmod 4$, whereas $d_{2}\left(v_{n}\right)$ satisfies $t+c \equiv 0 \bmod 4$. Hence $d_{2}\left(v_{n}\right)=h_{1}^{2} v_{n-1}^{2}$ is true on the nose. That the spectral sequence collapses at the $E_{3}$ page follows by degree reasons.

Theorem 9 For a field $F$ with $\operatorname{cd}_{2}(F) \leq 2$ and characteristic different from 2 the two-complete $\eta$-inverted Milnor-Witt stems of $F$ are

$$
\hat{\Pi}_{t}(F)\left[\eta^{-1}\right] \cong \begin{cases}W(F)_{2}^{\wedge}\left[\eta^{ \pm 1}\right] & t \geq 0 \text { and } t \equiv 3 \bmod 4 \text { or } t \equiv 0 \bmod 4 \\ 0 & \text { otherwise. }\end{cases}
$$

$\hat{\Pi}_{*}(F)\left[\eta^{-1}\right]$ is the polynomial ring over $W(F)_{2}^{\wedge}\left[\eta^{ \pm 1}\right]$ on two classes $\left\{v_{2}\right\}$ and $\{P\}$ in Milnor-Witt stem 3 and 4 respectively, subject to the relation $\left\{v_{2}\right\}^{2}=0$.

Proof $\hat{\Pi}_{0}(F)\left[\eta^{-1}\right]$ is shown to be $W(F)_{2}^{\wedge}\left[\eta^{ \pm 1}\right]$ in proposition ??. The remaining stems and ring structure follows from the calculation of the $h_{1}$-inverted motivic Adams spectral sequence over $F$ whose differentials are determined in proposition ??

We now identify some classes in $\pi_{* *}\left(\mathbb{1}_{2}^{\wedge}\right)\left(\mathbb{F}_{q}\right)$ for finite fields $\mathbb{F}_{q}$ using the analysis of the motivic Adams spectral sequence by Wilson and Østvær in [?]. Over a finite field $\mathbb{F}_{q}$ with $q \equiv 1 \bmod 4$, define $\varepsilon \in \pi_{8,5}\left(\mathbb{1}_{2}^{\wedge}\right) \cong(\mathbb{Z} / 2)^{4}$ to be a class detected by $c_{0}$. The class $\varepsilon$ is uniquely determined modulo $u \eta \varepsilon$. Write $\mu$ for a class in $\pi_{9,5}\left(\mathbb{1}_{2}^{\wedge}\right) \cong(\mathbb{Z} / 2)^{4}$ detected by $P h_{1}$. The class $\mu$ is uniquely determined modulo $u \eta \mu$.

Over a finite field $\mathbb{F}_{q}$ with $q \equiv 3 \bmod 4$, there is an isomorphism $\pi_{8,5}\left(\mathbb{1}_{2}^{\wedge}\right) \cong \mathbb{Z} / 4 \oplus \mathbb{Z} / 4$. Recall there is a Hopf map $\sigma \in \pi_{7,4}\left(\mathbb{1}_{2}^{\wedge}\right)$ defined by Dugger and Isaksen in [?]. The class $\eta \sigma$ generates an order four cyclic subgroup of $\pi_{8,5}\left(\mathbb{1}_{2}^{\wedge}\right)$; define $\varepsilon \in \pi_{8,5}\left(\mathbb{1}_{2}^{\wedge}\right)$ by the property that $\varepsilon$ generates $\pi_{8,5}\left(\mathbb{1}_{2}^{\wedge}\right) /(\eta \sigma)$. The class $\varepsilon$ is detected by $c_{0}$ and well defined up to an odd multiple. Further, there is an isomorphism $\pi_{9,5}\left(\mathbb{1}_{2}^{\wedge}\right) \cong \mathbb{Z} / 4 \oplus(\mathbb{Z} / 2)^{2}$. Define $\mu$ to be a class of order four that is detected by $P h_{1}$; the class $\mu$ is uniquely defined up to an odd multiple.

Corollary 10 For a finite field $\mathbb{F}_{q}$ with $q$ odd, the $\eta$-inverted Milnor-Witt stems are as follows.

$$
\hat{\Pi}_{n}\left(\mathbb{F}_{q}\right)\left[\eta^{-1}\right] \cong \begin{cases}W\left(\mathbb{F}_{q}\right)_{2}^{\wedge}\left[\eta^{ \pm 1}\right] & n \geq 0 \text { and } n \equiv 3 \bmod 4 \text { or } n \equiv 0 \bmod 4 \\ 0 & \text { otherwise }\end{cases}
$$


The classes $\mu$ and $\varepsilon$ generate $\hat{\Pi}_{*}\left(\mathbb{F}_{q}\right)\left[\eta^{-1}\right]$ as an algebra over $\hat{\Pi}_{0}\left(\mathbb{F}_{q}\right)\left[\eta^{-1}\right]$, subject to the relation $\varepsilon^{2}=0$. When $q \equiv 3 \bmod 4$ this shows $\hat{\Pi}_{*}\left(\mathbb{F}_{q}\right) \cong \mathbb{Z} / 4\left[\eta^{ \pm 1}, \mu, \epsilon\right] / \epsilon^{2}$ and for $q \equiv 1 \bmod 4$ we have $\hat{\Pi}_{*}\left(\mathbb{F}_{q}\right) \cong \mathbb{Z} / 2\left[\eta^{ \pm 1}, u, \mu, \epsilon\right] /\left(u^{2}, \epsilon^{2}\right)$.

Proof The mod 2 Milnor $K$-theory of a finite field with odd characteristic is given by $k_{*}^{M}\left(\mathbb{F}_{q}\right)=\mathbb{F}_{2}[u] / u^{2}$ where $u$ is the class of a non-square element of $\mathbb{F}_{q}^{\times}$. If $q \equiv 3 \bmod 4$ then $u=\rho=[-1]$. As $h_{1} \rho$ in $\mathfrak{M}\left(\mathbb{F}_{q}\right)\left[h_{1}^{-1}\right]$ detects multiplication by 2 in $\pi_{* *}\left(\mathbb{1}_{2}^{\wedge}\right)$, we arrive at the claimed group structure. The product structure is clear given the products in the $h_{1}$-inverted motivic Adams spectral sequence.

Corollary 11 The $\eta$-inverted Milnor-Witt stems for a $p$-adic field $\mathbb{Q}_{p}$ are as follows.

$$
\begin{gathered}
\hat{\Pi}_{0}\left(\mathbb{Q}_{p}\right)\left[\eta^{-1}\right] \cong W\left(\mathbb{Q}_{p}\right)_{2}^{\wedge}\left[\eta^{ \pm 1}\right] \cong \begin{cases}\mathbb{Z} / 2\left[\eta^{ \pm 1}, u, \pi\right] /\left(u^{2}, \pi^{2}\right) & p \equiv 1 \bmod 4 \\
(\mathbb{Z} / 4 \oplus \mathbb{Z} / 4)\left[\eta^{ \pm 1}\right] & p \equiv 3 \bmod 4 \\
(\mathbb{Z} / 8 \oplus \mathbb{Z} / 2 \oplus \mathbb{Z} / 2)\left[\eta^{ \pm 1}\right] & p=2\end{cases} \\
\hat{\Pi}_{n}\left(\mathbb{Q}_{p}\right)\left[\eta^{-1}\right] \cong \begin{cases}W\left(\mathbb{Q}_{p}\right)_{2}^{\wedge}\left[\eta^{ \pm 1}\right] & n \geq 0 \text { and } n \equiv 3 \bmod 4 \text { or } n \equiv 0 \bmod 4 \\
0 & \text { otherwise }\end{cases}
\end{gathered}
$$

Proof The mod 2 Milnor $K$-theory of the $p$-adic fields can be calculated from the result of Milnor [?, Lemma 4.6] in addition to the description of the Witt ring for $p$-adic fields which is discussed by Serre in [?]. Explicitly, the mod 2 Milnor $K$-theory of a $p$-adic field is

$$
k_{*}^{M}\left(\mathbb{Q}_{p}\right)= \begin{cases}\mathbb{Z} / 2[\pi, u] /\left(\pi^{2}, u^{2}\right) & p \equiv 1 \bmod 4 \\ \mathbb{Z} / 2[\pi, \rho] /\left(\rho^{2}, \rho \pi+\pi^{2}\right) & p \equiv 3 \bmod 4 \\ \mathbb{Z} / 2[\pi, \rho, u] /\left(\rho^{3}, u^{2}, \pi^{2}, \rho u, \rho \pi, \rho^{2}+u \pi\right) & p=2\end{cases}
$$

where $\pi=[p], \rho=[-1], u$ is the class of a lift of a non-square in $\mathbb{F}_{p}^{\times}$when $p \equiv 1 \bmod 4$, and $u=[5]$ when $p=2$.

\section{The field of rational numbers}

We approach the calculation of $\hat{\Pi}_{*}(\mathbb{Q})\left[\eta^{-1}\right]$ with the strategy suggested by the motivic Hasse principle, following the method of Ormsby and Østvær in [?]. That is, we analyze the $h_{1}$-inverted motivic Adams spectral sequence for $\mathbb{Q}$ using our knowledge of the $h_{1}$-inverted motivic Adams spectral sequence over the completions of $\mathbb{Q}$. 
We fix our notation for $k_{*}^{M}(\mathbb{Q})$. The $\bmod 2$ Milnor $K$-theory of $\mathbb{Q}$ is generated by the classes [-1] and $[p]$ for $p$ a prime. Milnor shows in [?, Lemma A.1] that there is a short exact sequence

$$
0 \rightarrow k_{2}^{M}(\mathbb{Q}) \rightarrow \bigoplus k_{2}^{M}\left(\mathbb{Q}_{\nu}\right) \rightarrow \mathbb{Z} / 2 \rightarrow 0
$$

where the summation is over all completions $\mathbb{Q}_{\nu}$ of $\mathbb{Q}$. For every completion $\mathbb{Q}_{\nu}$ of $\mathbb{Q}$ there is an isomorphism $k_{2}^{M}\left(\mathbb{Q}_{\nu}\right) \cong \mathbb{Z} / 2$; write $e_{\nu}$ for the image of 1 under the canonical map $k_{2}^{M}\left(\mathbb{Q}_{\nu}\right) \rightarrow \bigoplus k_{2}^{M}\left(\mathbb{Q}_{\nu}\right)$. For $\ell$ an odd prime or -1 , write $a_{\ell}$ for the class in $k_{2}^{M}(\mathbb{Q})$ that maps to $e_{\ell}+e_{2}$ in $\bigoplus k_{2}^{M}\left(\mathbb{Q}_{\nu}\right)$. For $n \geq 3$ the class $\rho^{n}$ generates $k_{n}^{M}(\mathbb{Q})$. The product structure in $k_{*}^{M}(\mathbb{Q})$ can be deduced from the products given in table ??; we write $\left(\frac{q}{\ell}\right)$ for the Legendre symbol that takes values in the additive group $\mathbb{Z} / 2$.

\begin{tabular}{l|l} 
Product & Conditions \\
\hline$[\ell][2]=0$ & $\ell=2$ or $\ell=-1$ \\
{$[-1][\ell]=a_{\ell}$} & $\ell=-1$ or $\ell$ prime and $\ell \equiv 3 \bmod 4$ \\
{$[-1][\ell]=0$} & $\ell$ prime and $\ell \equiv 1 \bmod 4$ \\
{$[\ell][q]=\left(\frac{q}{\ell}\right) a_{\ell}+\left(\frac{\ell}{q}\right) a_{q}$} & $\ell$ and $q$ odd primes \\
{$[2][q]=\left(\frac{q^{2}-1}{8} \bmod 2\right) a_{q}$} & $q$ odd prime
\end{tabular}

Table 1: Products in $k_{*}^{M}(\mathbb{Q})$

Lemma 12 The $E_{1}$ page of the $h_{1}$-inverted $\rho$-Bockstein spectral sequence over $\mathbb{Q}$ is the $\mathbb{Z} / 2$-algebra

$$
\mathfrak{B}(\mathbb{Q})\left[h_{1}^{-1}\right] \cong \bigoplus_{n \in \mathbb{N}} \rho^{n} k_{*}^{M}(\mathbb{Q}) / \rho^{n+1} \otimes_{\mathbb{F}_{2}} \operatorname{Ext}(\mathbb{C})\left[h_{1}^{-1}\right] .
$$

The class $\rho^{n}$ is in filtration $\epsilon=n$ for all $n \in \mathbb{N}$, for $\ell \equiv 3 \bmod 4$ a prime $a_{\ell}$ is in filtration 1 , for $\ell \equiv 1 \bmod 4$ a prime $a_{p}$ is in filtration 0 , and $[p]$ for $p$ a prime is in filtration 0 . The $r$ th differential $d_{r}$ for the $\rho$-Bockstein spectral sequence has degree $(\epsilon, f, t, c)=(r, 1,-1,0)$. See figure ?? for a chart of the $E_{1}$-page up to Milnor-Witt stem 15 .

Proof The $\rho$-Bockstein spectral sequence arises from filtering the cobar complex $\mathcal{C}^{*}(\mathbb{Q})$ by powers of $\rho$. The $s$ th term of the cobar complex is $\mathcal{C}^{s}(\mathbb{Q})=H_{* *}(\mathbb{Q}) \otimes \mathcal{A}_{* *}(\mathbb{Q})^{\otimes s}$, where the tensor products are taken over $H_{* *}(\mathbb{Q})$, taking care to use the left and right actions of $H_{* *}(\mathbb{Q})$ on $\mathcal{A}_{* *}(\mathbb{Q})$ arising from the left and right units $\eta_{L}$ and $\eta_{R}$. Any class $a\left[x_{1}|\cdots| x_{s}\right]$ can be reduced to a sum of monomials $b\left[y_{1}|\cdots| y_{s}\right]$ where each $y_{i}$ is a monomial in $\mathbb{Z} / 2\left[\tau_{0}, \tau_{1}, \ldots, \xi_{1}, \xi_{2}, \ldots\right]$. The class $\tau$ is killed after inverting $h_{1}$, hence every element of $\mathcal{C}^{s}(\mathbb{Q})$ is a sum of monomials $b\left[y_{1}|\cdots| y_{s}\right]$ where each $y_{i}$ is 
a monomial in $\mathbb{Z} / 2\left[\tau_{0}, \tau_{1}, \ldots, \xi_{1}, \xi_{2}, \ldots\right]$ and $b \in k_{*}^{M}(\mathbb{Q})$. The filtration of the cobar complex now is determined by the filtration of $k_{*}^{M}(\mathbb{Q})$ by powers of $\rho$.

Proposition 13 The differentials for the $h_{1}$-inverted $\rho$-Bockstein spectral sequence over $\mathbb{Q}$ are determined by $d_{2^{n}-1}\left(v_{1}^{2^{n}}\right)=h_{1}^{2^{n}} \rho^{2^{n}-1} v_{n}$ for $n \geq 2$ and $d_{r}\left(v_{n}\right)=0$ for $r \geq 1$ and $n \geq 2$. See figure ?? for a chart of the $E_{\infty}$ page up to Milnor-Witt stem 15 .

Proof The injection $k_{*}^{M}(\mathbb{Q}) \rightarrow \prod_{\nu} k_{*}^{M}\left(\mathbb{Q}_{\nu}\right)$ extends to an injection of $h_{1}$-inverted $\rho$-Bockstein spectral sequences at the $E_{1}$ page.

$$
\mathfrak{B}(\mathbb{Q})\left[h_{1}^{-1}\right] \rightarrow \prod_{\nu} \mathfrak{B}\left(\mathbb{Q}_{\nu}\right)\left[h_{1}^{-1}\right]
$$

The differentials in the $h_{1}$-inverted $\rho$-Bockstein spectral sequence over $\mathbb{Q}_{p}$ vanish for all primes $p$. Only the differentials in $\mathfrak{B}(\mathbb{R})\left[h_{1}^{-1}\right]$ contribute to the differentials over $\mathbb{Q}$, and these were identified by Guillou and Isaksen in [?, Lemma 3.1].

Proposition 14 The $h_{1}$-inverted $\rho$-Bockstein spectral sequence for $\mathbb{Q}$ converges strongly to $\operatorname{Ext}(\mathbb{Q})\left[h_{1}^{-1}\right]$ and there are no hidden extensions.

Proof The $h_{1}$-inverted $\rho$-Bockstein spectral sequence is isomorphic to the $\rho$-Bockstein spectral sequence obtained by filtering the $\left[\xi_{1}\right]$-inverted cobar complex $\mathcal{C}^{*}(\mathbb{Q})$, hence it converges strongly to $\operatorname{Ext}(\mathbb{Q})\left[h_{1}^{-1}\right]$. Guillou and Isaksen have shown that there are no hidden extensions in the $h_{1}$-inverted $\rho$-Bockstein spectral sequence over $\mathbb{R}\left[?\right.$, Proposition 4.9] and there are no hidden extensions in the $h_{1}$-inverted $\rho$ Bockstein spectral sequence over the other completions of $\mathbb{Q}$ by proposition ??. We therefore conclude there are no hidden extensions since the Hasse map embeds $\mathfrak{B}(\mathbb{Q})\left[h_{1}^{-1}\right]_{\infty} \Rightarrow \operatorname{Ext}(\mathbb{Q})\left[h_{1}^{-1}\right]$ into $\prod_{\nu} \mathfrak{B}\left(\mathbb{Q}_{\nu}\right)\left[h_{1}^{-1}\right]_{\infty} \Rightarrow \prod_{\nu} \operatorname{Ext}\left(\mathbb{Q}_{\nu}\right)\left[h_{1}^{-1}\right]$.

Corollary $15 \operatorname{Ext}(\mathbb{Q})\left[h_{1}^{-1}\right]$ is generated by the classes in table ??. The relations among these generators over $k_{*}^{M}(\mathbb{Q})$ include: $[\ell] P^{k} \cdot[q] P^{j}=[\ell] \cdot[q] P^{k+j}$ for $\ell, q$ primes and $k, j \geq 0 ;[\ell] P^{2^{n-1} k} \cdot v_{n}=[\ell] \cdot P^{2^{n-1} k} v_{n}$ for $\ell$ a prime, $n \geq 2$, and $k \geq 0$; the vanishing of the product of three or more generators of the form $[\ell] P^{k}$; and the relations which set the $\rho$-torsion of the generators.

Proof The generators can be determined by comparing $\operatorname{Ext}(\mathbb{Q})\left[h_{1}^{-1}\right]$ to $\operatorname{Ext}(\mathbb{R})\left[h_{1}^{-1}\right]$, and the latter was determined by Guillou and Isaksen in [?, Theorem 4.10]. The relations stated are present in the $\rho$-Bockstein spectral sequence and persist to $\operatorname{Ext}(\mathbb{Q})$. 


\begin{tabular}{c|c|c|l} 
Class & $(f, t, c)$ & $\rho$-torsion & Conditions \\
\hline$h_{1}^{ \pm 1}$ & $( \pm 1,0,0)$ & $\infty$ & \\
$\rho$ & $(0,0,1)$ & $\infty$ & \\
{$[2]+\rho$} & $(0,0,1)$ & 1 & \\
{$[\ell] P^{k}$} & $(0,0,1)+k(4,4,4)$ & 1 & $\ell$ prime, $\ell \equiv 1(4), k \geq 0$ \\
{$[\ell] P^{k}$} & $(0,0,1)+k(4,4,4)$ & 2 & $\ell$ prime, $p \equiv 3(4), k \geq 0$ \\
{$[2] P^{k}$} & $(0,0,1)+k(4,4,4)$ & 1 & $k \geq 1$ \\
$P^{2 k} v_{2}$ & $(1,3,1)+k(8,8,8)$ & 3 & $k \geq 0$ \\
$P^{4 k} v_{3}$ & $(1,7,1)+k(16,16,16)$ & 7 & $k \geq 0$ \\
$P^{8 k} v_{4}$ & $(1,15,1)+k(32,32,32)$ & 15 & $k \geq 0$ \\
$\ldots$ & $\ldots$ & $\cdots$ & $\cdots$
\end{tabular}

Table 2: Generators of $\operatorname{Ext}(\mathbb{Q})\left[h_{1}^{-1}\right]$.

The differentials in $\mathfrak{M}(\mathbb{Q})\left[h_{1}^{-1}\right]$, the $h_{1}$-inverted Adams spectral sequence over $\mathbb{Q}$, are determined by the differentials obtained from the comparison to $\mathbb{Q}_{p}$ and $\mathbb{R}$. See figures ?? and ?? for a depiction of the $E_{2}$ and $E_{3}$ pages up to Milnor-Witt stem 15.

Proposition 16 The $d_{2}$ differential in $\mathfrak{M}(\mathbb{Q})\left[h_{1}^{-1}\right]$ is determined by the Leibniz rule from the equations $d_{2}\left(P^{2^{n-1}} k_{n}\right)=P^{2^{n-1}} v_{n-1}^{2}$ for $k \geq 0$ and $n \geq 3$ and the vanishing of $d_{2}$ on the remaining generators. For $r \geq 3$, the differential $d_{r}$ in $\mathfrak{M}(\mathbb{Q})\left[h_{1}^{-1}\right]$ is determined by the Leibniz rule from the equations

$$
d_{r}\left(\rho^{2^{n}-2^{n-r+2}-r+2} P^{2^{n-1}} k v_{n}\right)=P^{2^{n-1} k+2^{n-2}-2^{n-r}} v_{n-r+1}^{2}
$$

for $n \geq r+1$ and the vanishing of $d_{r}$ on the remaining generators.

Proof $\operatorname{Ext}(\mathbb{Q})\left[h_{1}^{-1}\right]$ injects into the product $\prod_{\nu} \operatorname{Ext}\left(\mathbb{Q}_{\nu}\right)\left[h_{1}^{-1}\right]$ under the base change maps obtained from $\mathbb{Q} \rightarrow \mathbb{Q}_{\nu}$. The map is seen to be injective by the explicit calculation of $\operatorname{Ext}(\mathbb{Q})\left[h_{1}^{-1}\right]$ given in corollary ??, $\operatorname{Ext}\left(\mathbb{Q}_{p}\right)\left[h_{1}^{-1}\right]$ in proposition ??, and $\operatorname{Ext}(\mathbb{R})\left[h_{1}^{-1}\right]$ in [?, Theorem 4.10]. The differentials $d_{2}\left(v_{n}\right)=v_{n-1}^{2}$ for $n \geq 3$ over $\mathbb{Q}_{p}$ imply that the class $d_{2}\left(P^{2^{n-1}} k_{n}\right)$ must map to $d_{2}\left(P^{2^{n-1} k} v_{n}\right)=P^{2^{n-1} k} v_{n-1}^{2}$ in $\operatorname{Ext}\left(\mathbb{Q}_{p}\right)\left[h_{1}^{-1}\right]$. Comparison to $\mathbb{R}$ also shows that the differential $d_{2}\left(P^{2^{n-1}} v_{n}\right)$ maps to $P^{2^{n-1}} v_{n-1}^{2}$ in $\operatorname{Ext}(\mathbb{R})\left[h_{1}^{-1}\right]$ for $n \geq 3$, as determined by Guillou and Isaksen [?, Lemma 5.2]. The differential $d_{2}$ over $\mathbb{Q}$ vanishes on the classes $[\ell] P^{k}$ by comparison to $\mathbb{Q}_{p}$ for all $p$. Finally, $d_{2}$ vanishes on all elements of $k_{*}^{M}(\mathbb{Q})$ for degree reasons. This accounts for all of irreducible classes of $\operatorname{Ext}(\mathbb{Q})\left[h_{1}^{-1}\right]$; the generators for $\mathfrak{M}(\mathbb{Q})_{3}\left[h_{1}^{-1}\right]$ are given in table ??. Note that the classes $P^{2(2 j+1)} v_{2}^{2}$ also survive but decompose as the product $P^{2(2 j+1)} v_{2} \cdot v_{2}$. 


\begin{tabular}{c|c|c|l} 
Class & $(f, t, c)$ & $\rho$-torsion & Conditions \\
\hline$h_{1}^{ \pm 1}$ & $( \pm 1,0,0)$ & $\infty$ & \\
$\rho$ & $(0,0,1)$ & $\infty$ & \\
{$[2]+\rho$} & $(0,0,1)$ & 1 & \\
{$[\ell] P^{k}$} & $(0,0,1)+k(4,4,4)$ & 1 & $\ell$ prime, $\ell \equiv 1(4), k \geq 0$ \\
{$[\ell] P^{k}$} & $(0,0,1)+k(4,4,4)$ & 2 & $\ell$ prime, $\ell \equiv 3(4), k \geq 0$ \\
{$[2] P^{k}$} & $(0,0,1)+k(4,4,4)$ & 1 & $k \geq 1$ \\
$P^{2 k} v_{2}$ & $(1,3,1)+k(8,8,8)$ & 3 & $k \geq 0$ \\
$\rho^{3} P^{4 k} v_{3}$ & $(1,7,4)+k(16,16,16)$ & 4 & $k \geq 0$ \\
$\rho^{7} P^{8 k} v_{4}$ & $(1,15,8)+k(32,32,32)$ & 8 & $k \geq 0$ \\
$\ldots$ & $\cdots$ & $\cdots$ & $\cdots$ \\
$P^{4(2 j+1)} v_{3}^{2}$ & $(2,14,2)+(2 j+1)(4,4,4)$ & 7 & $j \geq 0$ \\
$P^{8(2 j+1)} v_{4}^{2}$ & $(2,30,2)+(2 j+1)(4,4,4)$ & 15 & $j \geq 0$ \\
$\ldots$ & $\cdots$ & $\cdots$ & $\cdots$
\end{tabular}

Table 3: Generators of $\mathfrak{M}(\mathbb{Q})\left[h_{1}^{-1}\right]_{3}$.

The Hasse map

$$
\mathfrak{M}(\mathbb{Q})_{3}\left[h_{1}^{-1}\right] \rightarrow \prod_{\nu} \mathfrak{M}\left(\mathbb{Q}_{\nu}\right)_{3}\left[h_{1}^{-1}\right]
$$

is still injective. Over the $p$-adic fields, all further differentials vanish, and over $\mathbb{R}$ the differentials are determined by Guillou and Isaksen [?, Lemma 5.8]; these comparisons determine the remaining differentials.

Proposition $17 \mathfrak{M}(\mathbb{Q})\left[h_{1}^{-1}\right]_{\infty}$ is generated over $k_{*}^{M}(\mathbb{Q})$ by the classes $\rho^{2^{n}-n-2} P^{2^{n-1}} k_{n}$ for $k \geq 0$ and $n \geq 2$, which has degree $\left(2^{n+1} k+1,2^{n+1} k+2^{n}-1,2^{n+1} k+2^{n}-n-1\right)$ and $\rho$-torsion $n+1$. See table ?? for some low degree generators and figure ?? for a chart of the $E_{\infty}$ page up to Milnor-Witt stem 15 .

Proof This is a consequence of the differential analysis of proposition ?? and the result of Guillou and Isaksen [?, Proposition 5.9].

Definition 18 Let $M$ be the submodule of $W(\mathbb{Q})_{2}^{\wedge}\left[\eta^{ \pm 1}\right]$ generated by the rank one forms $\ell \cdot X^{2}$ for $\ell$ a prime. As an abelian group, $M$ is isomorphic to $\mathbb{Z} / 2 \oplus \bigoplus_{p \equiv 3 \text { (4) }} \mathbb{Z} / 4 \oplus$ $\bigoplus_{p \equiv 1(4)}(\mathbb{Z} / 2)^{2}\left[\eta^{ \pm 1}\right]$.

Following the notational convention of Isaksen and Guillou [?, §7], write $P^{2^{n-1} k} \lambda_{n}$ for a class in $\hat{\Pi}_{t}(\mathbb{Q})\left[\eta^{-1}\right]$ detected by $\rho^{2^{n}-n-2} P^{2^{n-1}} k_{n}$ in $\mathfrak{M}(\mathbb{Q})\left[h_{1}^{-1}\right]$ where $n \geq 2$, 


\begin{tabular}{c|c|c|c} 
Class & $(f, t, c)$ & $\rho$-torsion & Conditions \\
\hline$h_{1}^{ \pm 1}$ & $( \pm 1,0,0)$ & $\infty$ & \\
$\rho$ & $(0,0,1)$ & $\infty$ & \\
{$[2]+\rho$} & $(0,0,1)$ & 1 & \\
{$[\ell] P^{k}$} & $(0,0,1)+k(4,4,4)$ & 1 & $\ell$ prime, $\ell \equiv 1(4), k \geq 0$ \\
{$[\ell] P^{k}$} & $(0,0,1)+k(4,4,4)$ & 2 & $\ell$ prime, $\ell \equiv 3(4), k \geq 0$ \\
{$[2] P^{k}$} & $(0,0,1)+k(4,4,4)$ & 1 & $k \geq 1$ \\
$P^{2 k} v_{2}$ & $(1,3,1)+k(8,8,8)$ & 3 & $k \geq 0$ \\
$\rho^{3} P^{4 k} v_{3}$ & $(1,7,4)+k(16,16,16)$ & 4 & $k \geq 0$ \\
$\rho^{10} P^{8 k} v_{4}$ & $(1,15,11)+k(32,32,32)$ & 5 & $k \geq 0$ \\
$\cdots$ & $\cdots$ & $\cdots$ & $\cdots$
\end{tabular}

Table 4: Generators of $\mathfrak{M}(\mathbb{Q})\left[h_{1}^{-1}\right]_{\infty}$.

$k \geq 0$ and $t=2^{n+1} k+2^{n}-1$. Also, we abuse notation and write $[\ell] P^{k}$ for a class in $\hat{\Pi}_{4 k}(\mathbb{Q})\left[\eta^{-1}\right]$ detected by the class of the same name in $\mathfrak{M}(\mathbb{Q})\left[h_{1}^{-1}\right]$.

Theorem 19 The $\eta$-inverted Milnor-Witt 0 stem of $\mathbb{1}_{2}^{\wedge}$ over $\mathbb{Q}$ is

$$
\hat{\Pi}_{0}(\mathbb{Q})\left[\eta^{-1}\right] \cong W(\mathbb{Q})_{2}^{\wedge}\left[\eta^{ \pm 1}\right] .
$$

The $t$ th $\eta$-inverted Milnor-Witt stem of $\mathbb{1}_{2}^{\wedge}$ over $\mathbb{Q}$ are as follows.

$$
\hat{\Pi}_{t}(\mathbb{Q})\left[\eta^{-1}\right] \cong \begin{cases}\hat{\Pi}_{0}(\mathbb{Q})\left[\eta^{-1}\right] / 2^{n+1} & t \geq 0, t \equiv 3 \bmod 4, n=\nu_{2}(t+1) \\ M & t \equiv 0 \bmod 4, t \geq 4 \\ 0 & \text { otherwise }\end{cases}
$$

Here $\nu_{2}(x)$ is the 2 -adic valuation of an integer $x$ and $M$ is the $\hat{\Pi}_{0}(\mathbb{Q})\left[\eta^{-1}\right]$-module of definition ??.

The remaining product structure of $\hat{\Pi}_{*}(\mathbb{Q})\left[\eta^{-1}\right]$ is determined by the following relations: the product of any two generators with Milnor-Witt stem congruent to $3 \bmod 4$ is zero; $[q] P^{j} \cdot[\ell] P^{k}=[q] \cdot[\ell] P^{j+k}$ for all primes $\ell$ and $q$ and $k, j \geq 0 ;[q] \cdot[\ell] P^{k}=0$ if $q$ is a prime or -1 and $[q][\ell]=0$ in $k_{*}^{M}(\mathbb{Q})$.

Proof The zero stem was calculated in proposition ?? and [?, Proof of Theorem 1.5] shows the one stem vanishes. Proposition ?? identifies the structure of the $E_{\infty}$ page of the $h_{1}$-inverted motivic Adams spectral sequence over $\mathbb{Q}$ and corollary ?? shows that $\mathfrak{M}(\mathbb{Q})\left[h_{1}^{-1}\right]$ strongly converges to $\hat{\Pi}_{*}(\mathbb{Q})\left[\eta^{-1}\right]$ in Milnor-Witt stem at least two. The 2 -extensions are resolved because $\rho h_{1}$ detects multiplication by 2 , from which the additive structure of the $\eta$-inverted stems follows. 
The product structure in the $E_{\infty}$ page of the $h_{1}$-inverted motivic Adams spectral sequence determines the $\hat{\Pi}_{0}(\mathbb{Q})\left[\eta^{-1}\right]$-module structure of the stems $\hat{\Pi}_{t}(\mathbb{Q})\left[\eta^{-1}\right]$ for $t \not \equiv 3 \bmod 4$ and $t \equiv 3 \bmod 8$. It only remains to identify the hidden product of $[\ell]$ for $\ell$ a prime with a class $P^{2^{n-1} k} \lambda_{n}$ of $\hat{\Pi}_{t}(\mathbb{Q})\left[\eta^{-1}\right]$ for $u \geq 3, k \geq 0$; note that $n=\nu_{2}(t+1)$. Lemma ?? shows that the products $[\ell] \cdot P^{2^{n-1} k} \lambda_{n}$ and $a_{\ell} \cdot P^{2^{n-1} k} \lambda_{n}$ are always non-zero; hence the canonical map $\hat{\Pi}_{0}(\mathbb{Q})\left[\eta^{-1}\right] / 2^{n+1} \rightarrow \hat{\Pi}_{n}(\mathbb{Q})\left[\eta^{-1}\right]$ is an isomorphism.

The product of any two generators with Milnor-Witt stem congruent to $3 \mathrm{mod} 4$ is zero for degree reasons. The remaining products are detected in the motivic Adams spectral sequence.

Lemma 20 For $n \geq 3$ and $k \geq 0$, the products $[\ell] \cdot P^{2^{n-1} k} \lambda_{n}$ with $\ell$ a prime and $a_{\ell} \cdot P^{2^{n-1} k} \lambda_{n}$ with $\ell$ an odd prime in $\hat{\Pi}_{*}(\mathbb{Q})\left[\eta^{-1}\right]$ are non-zero.

Proof For $m \geq 0$ and $\ell$ a prime, the Massey product $\left\langle\rho P^{2 m} v_{2}, \rho^{2},[\ell]\right\rangle$ in $\operatorname{Ext}(\mathbb{Q})\left[h_{1}^{-1}\right]$ contains $[\ell] P^{2 m+1}$ by lemma ?? and this has no indeterminacy. The hypotheses of Moss's convergence theorem [?, Theorem 3.1.1] hold in this case $;^{1}$ hence $[\ell] P^{2 m+1}$ detects a class of $\left\langle 2 P^{2 m} \lambda_{2}, 2^{2},[\ell]\right\rangle$. The indeterminacy of this Toda bracket is $[\ell] \hat{\Pi}_{8 m+4}$, which is in higher filtration than $[\ell] P^{2 m+1}$. We conclude $\left\langle 2 P^{2 m} \lambda_{2}, 2^{2},[\ell]\right\rangle$ does not contain zero.

The Massey product $\left\langle v_{2}, \rho P^{2 m} v_{2}, \rho^{2}\right\rangle$ can be shown to contain $\rho^{2^{n}-n-2} P^{2^{n-1}} k_{n}$ using the Adams differential

$$
d_{r}\left(\rho^{2^{n}-n-4} P^{2^{n-1} k} v_{n}\right)=\rho P^{2 m} v_{2}^{2}
$$

where $n=\nu_{2}(m+1)+3$ and $r=n-1$. This Massey product has trivial indeterminacy. Moss's convergence theorem shows that $\rho^{2^{n}-n-2} P^{2^{n-1}} k_{n}$ detects a class of $\left\langle\lambda_{2}, 2 P^{2 m} \lambda_{2}, 2^{2}\right\rangle ;^{2}$ hence $P^{2^{n-1} k} \lambda_{n}$ is in the Toda bracket $\left\langle\lambda_{2}, 2 P^{2 m} \lambda_{2}, 2^{2}\right\rangle$.

We now use the shuffle relation

$$
\lambda_{2}\left\langle 2 P^{2 m} \lambda_{2}, 2^{2},[\ell]\right\rangle=\left\langle\lambda_{2}, 2 P^{2 m} \lambda_{2}, 2^{2}\right\rangle[\ell] .
$$

\footnotetext{
${ }^{1}$ Observe that for $r \geq 2, c>t$ and $t \equiv 3 \bmod 4$ that the groups $E_{2}^{t, c}$ are trivial in the $h_{1}$-inverted motivic Adams spectral sequence over $\mathbb{Q}$.

${ }^{2}$ The condition to check for $\rho P^{2 m} v_{2}^{2}$ in Moss's theorem is that $d_{r^{\prime}}: E_{r^{\prime}}^{8 m+7, c^{\prime}} \rightarrow E_{r^{\prime}}^{8 m+6, c^{\prime \prime}}$ must be zero when $c^{\prime} \leq 8 m+1-\nu_{2}(m+1), c^{\prime \prime} \geq 8 m+4$ and $r^{\prime}=c^{\prime \prime}-c^{\prime}$. Nonzero differentials are only possible in these Milnor-Witt stems on classes $\rho^{?} P^{?} v_{n}$; it follows that $\nu_{2}(m+1)=n-3$, so $r^{\prime} \geq n$. But by proposition ??, nonzero differentials on such classes occur only when $n \geq r^{\prime}+1$. The condition to check for the element $\rho^{3} P^{2 m} v_{2}$ is that $d_{r^{\prime}}: E_{r^{\prime}}^{8 m+4, c^{\prime}} \rightarrow E_{r^{\prime}}^{8 m+3, c^{\prime \prime}}$ must be zero when $c^{\prime} \leq 8 m+2-\nu_{2}(m+1), c^{\prime \prime} \geq 8 m+5$ and $r^{\prime}=c^{\prime \prime}-c^{\prime}$. The classes in Milnor-Witt stem $4 \bmod 8$ are generated by the classes of the form $\rho, h_{1}^{ \pm 1}$ and $[\ell] P^{k}$. The Adams differentials vanish on these classes, so the hypotheses of Moss's theorem are true.
} 
Multiplication by $\lambda_{2}$ is an injection on the stems $\hat{\Pi}_{4 j}(\mathbb{Q})\left[\eta^{-1}\right] \rightarrow \hat{\Pi}_{4 j+3}(\mathbb{Q})\left[\eta^{-1}\right]$ by the product structure in the motivic Adams spectral sequence, hence the left hand side of the shuffle relation does not contain zero. As $[\ell] \cdot P^{2^{n-1} k} \lambda_{n}$ is in the right hand side of the shuffle relation, we conclude that $[\ell] \cdot P^{2^{n-1} k} \lambda_{n}$ is nonzero.

A similar argument using the shuffle relation

$$
\lambda_{2}\left\langle 2 P^{2 k} v_{2}, 2^{2}, a_{\ell}\right\rangle=\left\langle\lambda_{2}, 2 P^{2 k} v_{2}, 2^{2}\right\rangle a_{\ell}
$$

establishes the claim that $a_{\ell} \cdot P^{2^{u-1} k}$ is non-zero.

Lemma 21 Let $m \geq 0$ and $\ell$ a prime, the Massey product $\left\langle\rho P^{2 m} v_{2}, \rho^{2},[\ell]\right\rangle$ in $\operatorname{Ext}(\mathbb{Q})\left[h_{1}^{-1}\right]$ contains $[\ell] P^{2 m+1}$ and has trivial indeterminacy.

Proof The $\rho$-Bockstein spectral sequence differential $d_{3}\left(P^{2 m+1}\right)=\rho^{3} P^{2 m} v_{2}$ shows that $\left\langle\rho P^{2 m} v_{2}, \rho^{2},[\ell]\right\rangle$ contains $[\ell] P^{2 m+1}$; this Massey product has trivial indeterminacy. To verify the hypotheses of May's convergence theorem [?, Theorem 2.2.1], first note the degree of $\rho^{2}[\ell]$ is $\epsilon=2, t=0$, and $c=1$. All $\rho$-Bockstein spectral sequence differentials vanish in this graded component. It remains to check $d_{R}$ differentials on the graded piece with $\epsilon^{\prime} \geq 3, t=8 m+3, c=8 m+4$ and $R>\epsilon^{\prime}$ corresponding to $\rho^{3} P^{2 m} v_{2}$.

We now look for elements of the $E_{4}$-page of the $\rho$-Bockstein spectral sequence which land in degrees $(t, c)$ for which $t+c \equiv 7 \bmod 8$. Given the description of the generators of the $E_{4}$-page, it suffices to consider products of just $v_{n}$ for $n \geq 3,[\ell] P, v_{2}$, and $\rho$. The sum $t+c$ mod 8 for each of these generators is $0,1,4$, and 1 respectively. As $([\ell] P)^{2}=0, \rho^{3} v_{2}=0$, and $\rho^{2}[\ell] P v_{2}=0$ in the $E_{4}$-page, the only non-zero product of these generators in degree $(t, c)$ with $t+c \equiv 7 \bmod 8$ must be of the form $\rho^{\epsilon} v_{3}^{a_{3}} \cdots v_{i}^{a_{i}}$.

Suppose now that $\rho^{\epsilon} v_{3}^{a_{3}} \cdots v_{i}^{a_{i}}$ is in degree $t=8 m+3, c=8 m+4$ for some $m$. Let $A=\sum a_{i}$ and $j=\min \left\{x \mid a_{x} \neq 0\right\}$. Under these assumptions it follows that

$$
8 m+3=\sum a_{i}\left(2^{i}-1\right) \geq A\left(2^{j}-1\right) .
$$

Hence $\epsilon \geq A\left(2^{j}-2\right)+1$. Note that $A$ must be at least 2 in order for the Milnor-Witt stem $t$ to be congruent to 3 modulo 8 . It follows that if $R>\epsilon$, then $R>2^{j}-1$ and so the class $\rho^{\epsilon} v_{3}^{a_{3}} \cdots v_{i}^{a_{i}}$ is zero in the $E_{R}$ page due to the relation $\rho^{2^{j}-1} v_{j}=0$, which arises from a $d_{2^{j}-1}$ differential. We conclude May's convergence theorem applies in this situation. It is straightforward to check that the indeterminacy is trivial. 


\section{References}

[1] A Ananyevskiy, M Levine, I Panin, Witt sheaves and the $\eta$-inverted sphere spectrum, J. Topol. 10 (2017) 370-385

[2] M Andrews, H Miller, Inverting the Hopf map, preprint (2016)

[3] J M Boardman, Conditionally convergent spectral sequences, from: "Homotopy invariant algebraic structures (Baltimore, MD, 1998)", Contemp. Math. 239, Amer. Math. Soc., Providence, RI (1999) 49-84

[4] A K Bousfield, The localization of spectra with respect to homology, Topology 18 (1979) 257-281

[5] A K Bousfield, D M Kan, Homotopy limits, completions and localizations, Lecture Notes in Mathematics, Vol. 304, Springer-Verlag, Berlin-New York (1972)

[6] D Dugger, D C Isaksen, Motivic Hopf elements and relations, New York J. Math. 19 (2013) 823-871

[7] B J Guillou, D C Isaksen, The $\eta$-local motivic sphere, J. Pure Appl. Algebra 219 (2015) 4728-4756

[8] B J Guillou, D C Isaksen, The $\eta$-inverted $\mathbb{R}$-motivic sphere, Algebr. Geom. Topol. 16 (2016) 3005-3027

[9] P Hu, I Kriz, K Ormsby, Convergence of the motivic Adams spectral sequence, J. K-Theory 7 (2011) 573-596

[10] D C Isaksen, Classical and motivic Adams charts, arXiv:1401.4983 (2014)

[11] D C Isaksen, Stable stems, arXiv:1407.8418 (2014)

[12] M Knebusch, Symmetric bilinear forms over algebraic varieties (1977) 103-283. Queen's Papers in Pure and Appl. Math., No. 46

[13] J Milnor, Algebraic K-theory and quadratic forms, Invent. Math. 9 (1969/1970) 318-344

[14] F Morel, $\mathbb{A}^{1}$-algebraic topology over a field, volume 2052 of Lecture Notes in Mathematics, Springer, Heidelberg (2012)

[15] K M Ormsby, P A Østvær, Motivic Brown-Peterson invariants of the rationals, Geom. Topol. 17 (2013) 1671-1706

[16] K M Ormsby, O Röndigs, P A Østvær, Vanishing in stable motivic homotopy sheaves, arxiv:1704.04744v1 (2017)

[17] W Scharlau, Quadratic and Hermitian forms, volume 270 of Grundlehren der Mathematischen Wissenschaften, Springer-Verlag, Berlin (1985)

[18] J-P Serre, A course in arithmetic, Springer-Verlag, New York-Heidelberg (1973)Translated from the French, Graduate Texts in Mathematics, No. 7 
[19] C A Weibel, An introduction to homological algebra, volume 38 of Cambridge Studies in Advanced Mathematics, Cambridge University Press, Cambridge (1994)

[20] G M Wilson, P A Østvær, Two-complete stable motivic stems over finite fields, Algebr. Geom. Topol. 17 (2017) 1059-1104

Department of Mathematics, University of Oslo, Norway.

glenw@math.uio.no 


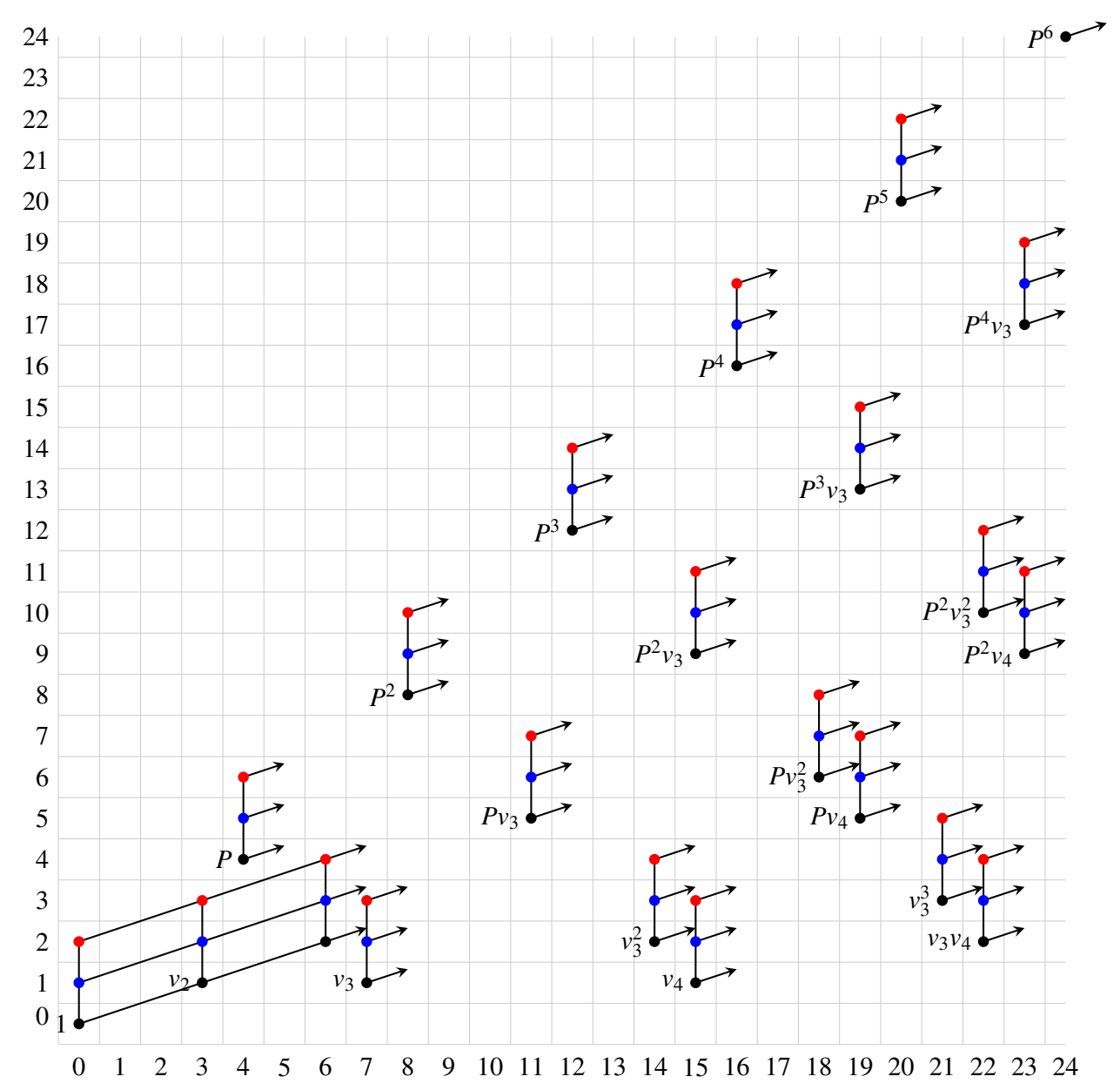

Figure 1: The $E_{1}$ page of the $h_{1}$-inverted $\rho$-Bockstein spectral sequence over a field $F$ with $\operatorname{cd}_{2}(F)=2$ up to Milnor-Witt stem 24. Solid vertical lines indicate possible $\rho$-multiplications that depend on the field. Black dots represent the group $\mathbb{Z} / 2\left[h_{1}^{ \pm 1}\right]$, blue dots represent $k_{1}^{M}(F)\left[h_{1}^{ \pm 1}\right]$, and red dots represent $k_{2}^{M}(F)\left[h_{1}^{ \pm 1}\right]$. Solid lines of slope $1 / 3$ indicate multiplication by $v_{2}$ and arrows in this direction represent a tower of nonzero $v_{2}$ multiples. The horizontal axis $t$ is the Milnor-Witt stem and the vertical axis $c$ is the Chow weight, while the Adams filtration is suppressed. 


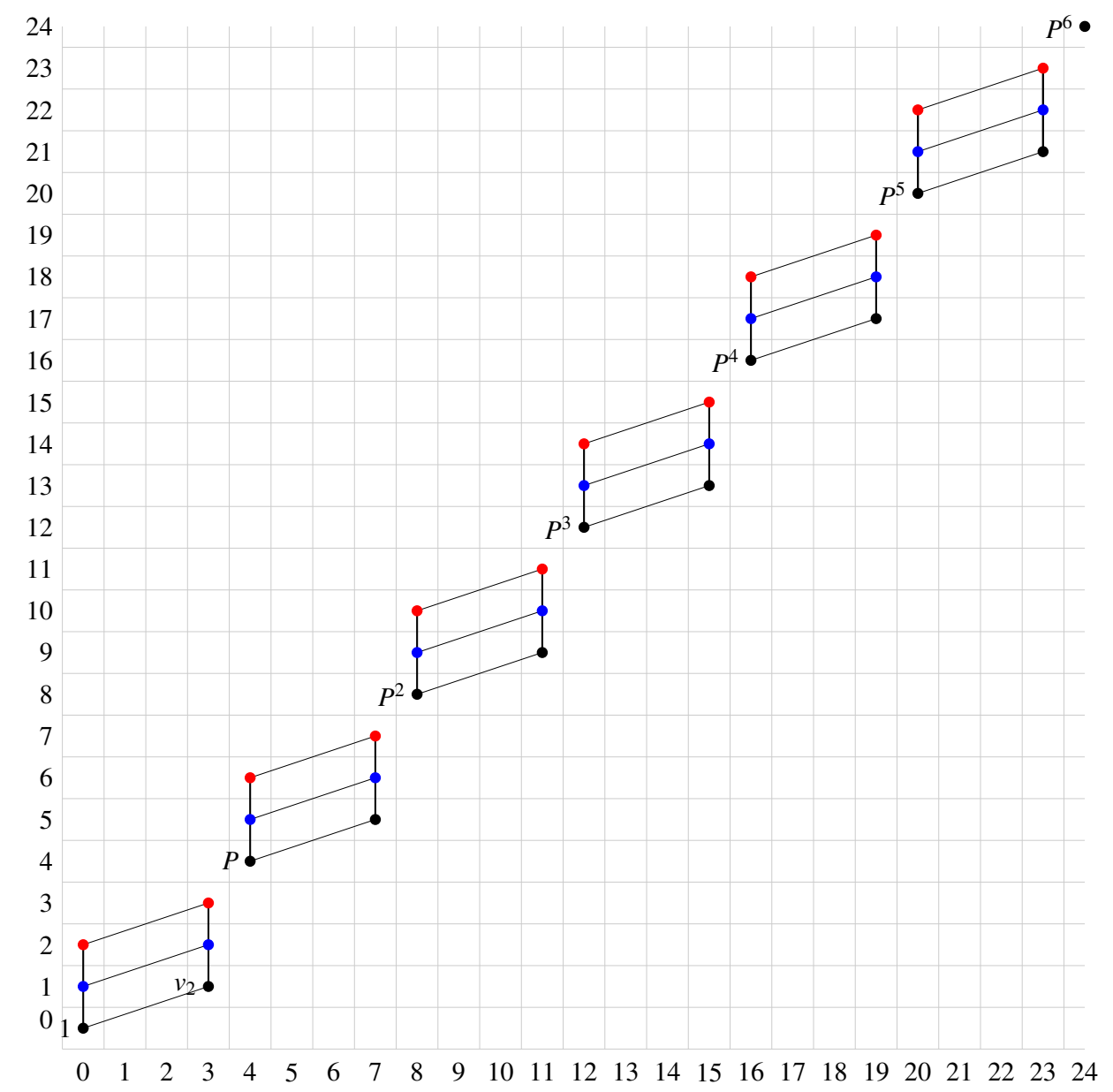

Figure 2: The $E_{\infty}$ page of the $h_{1}$-inverted motivic Adams spectral sequence over a field $F$ with $\operatorname{cd}_{2}(F) \leq 2$ up to Milnor-Witt stem 24. The notational conventions of figure ?? apply here. 


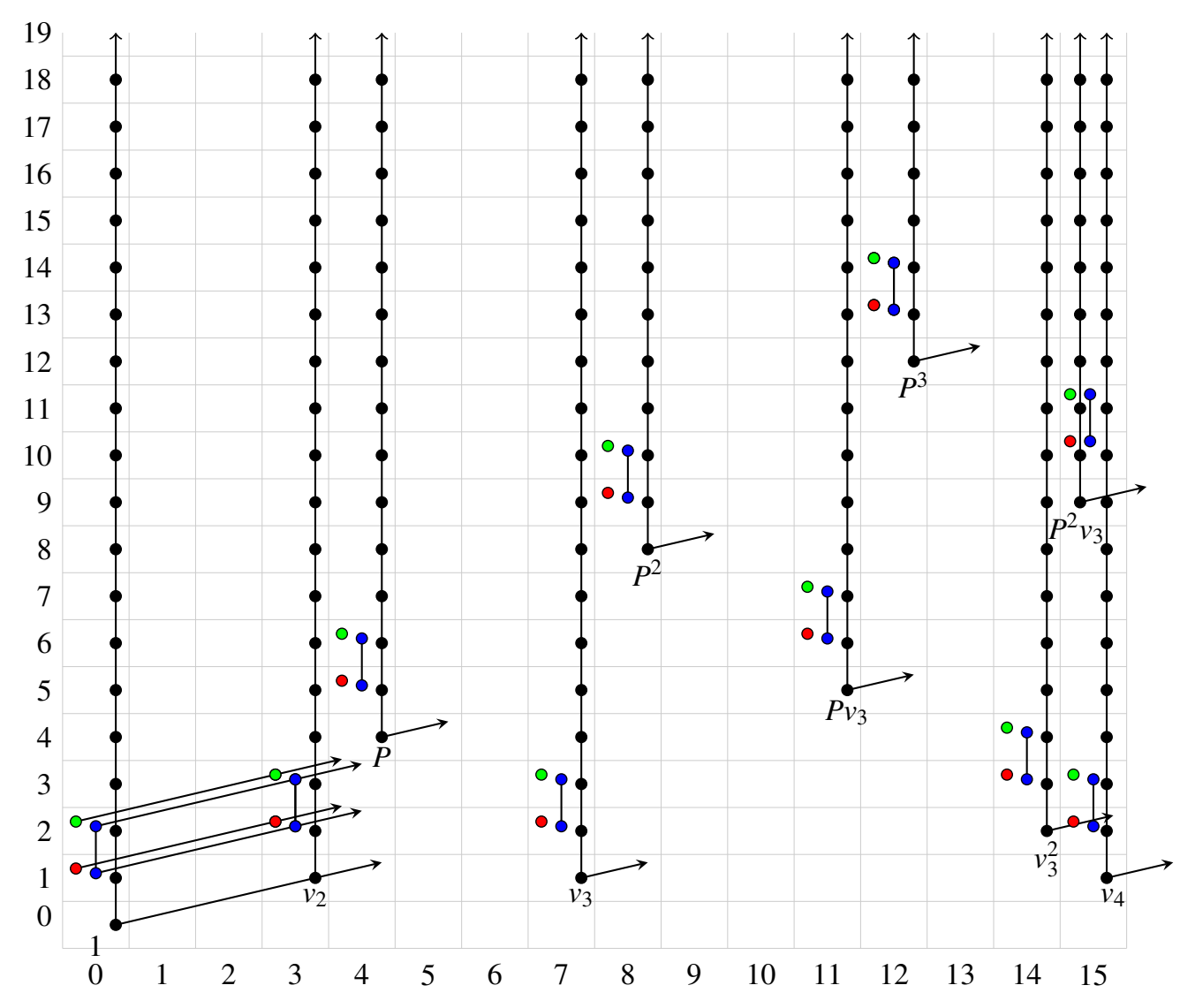

Figure 3: The $E_{1}$ page of the $h_{1}$-inverted $\rho$-Bockstein spectral sequence over $\mathbb{Q}$ up to MilnorWitt stem 15 . Black dots represent the group $\mathbb{Z} / 2\left[h_{1}^{ \pm 1}\right]$, blue dots represent $\oplus_{p \equiv 3(4)} \mathbb{Z} / 2\left[h_{1}^{ \pm 1}\right]$, red dots represent $\oplus_{p \equiv 1(4), p=2} \mathbb{Z} / 2\left[h_{1}^{ \pm 1}\right]$, and green dots represent $\oplus_{p \equiv 1(4)} \mathbb{Z} / 2\left[h_{1}^{ \pm 1}\right]$. Solid vertical lines indicate multiplication by $\rho$ and a vertical arrow means that the tower of $\rho$ multiplications continues indefinitely. Every dot supports an infinite tower of $v_{2}$-multiples, however we only indicate this with lines and arrows of slope $1 / 3$ on the classes of $\operatorname{Ext}(\mathbb{C})\left[h_{1}^{-1}\right]$ and $k_{*}^{M}(\mathbb{Q})$. The horizontal axis $t$ is the Milnor-Witt stem and the vertical axis $c$ is the Chow weight, while the Adams filtration is suppressed. 
Glen Matthew Wilson

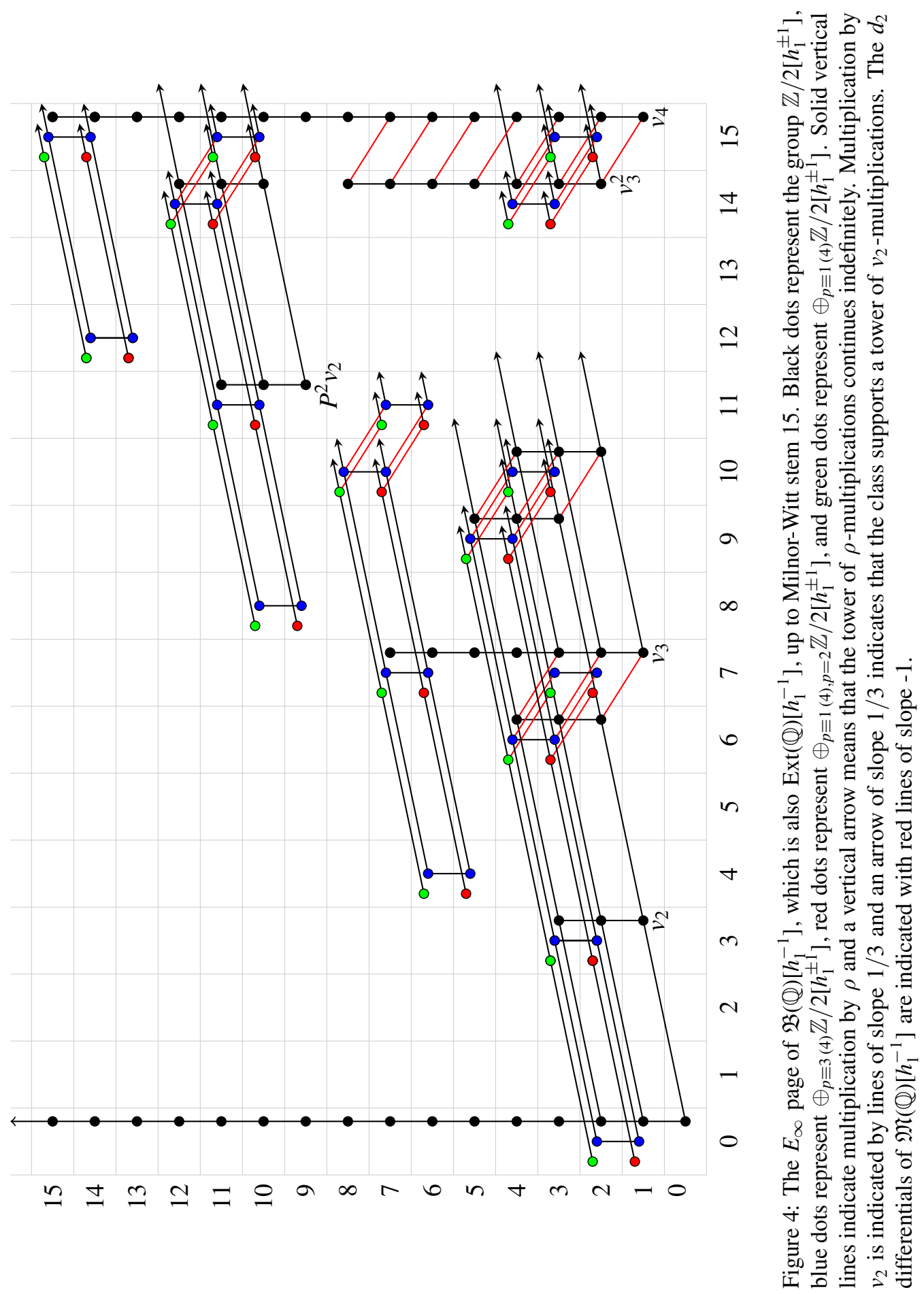




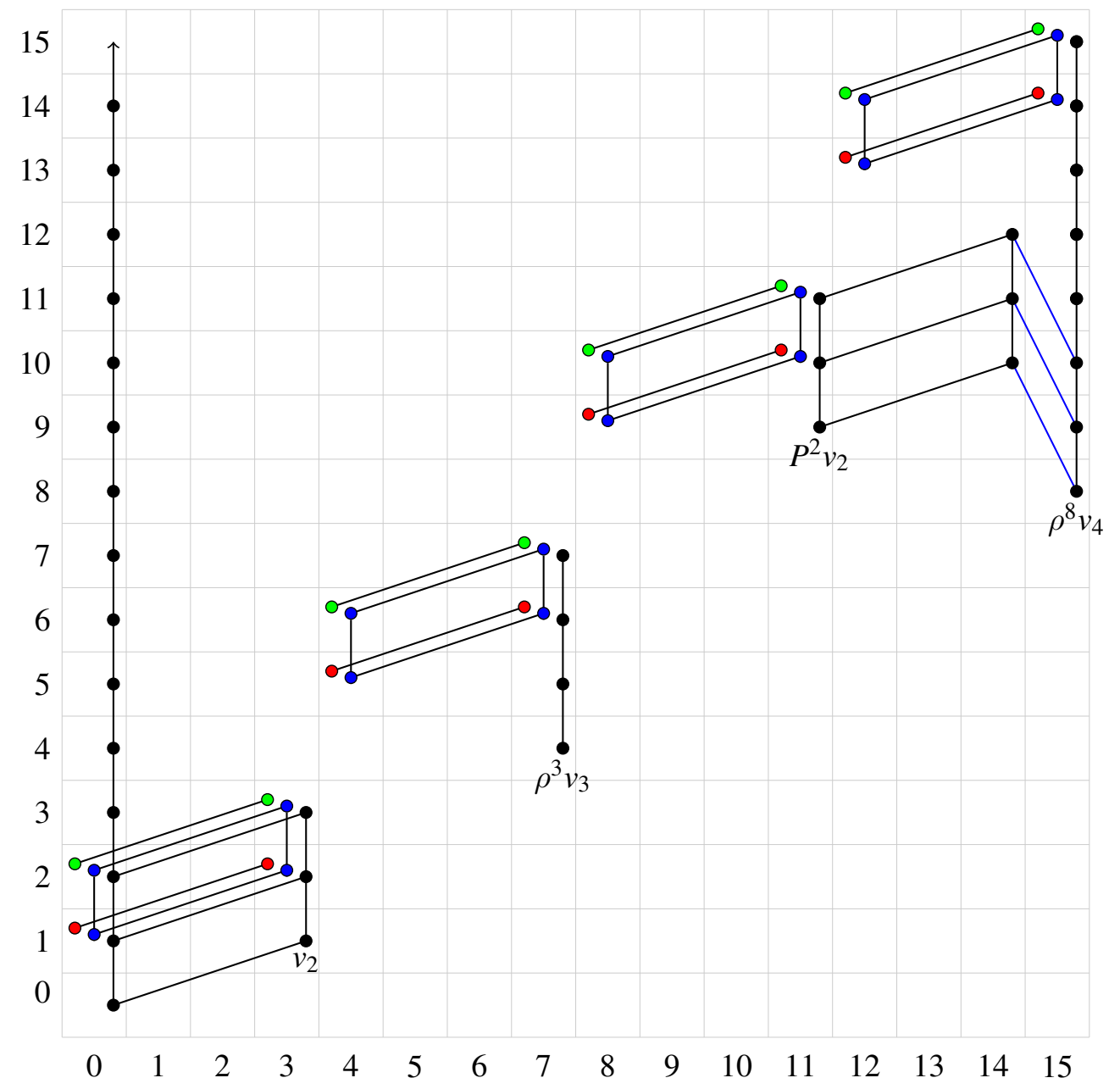

Figure 5: The $E_{3}$ page of $\mathfrak{M}(\mathbb{Q})\left[h_{1}^{-1}\right]$ up to Milnor-Witt stem 15 . The $d_{3}$ differentials are indicated with blue lines of slope -2 . The notational conventions of figure ?? apply here. 


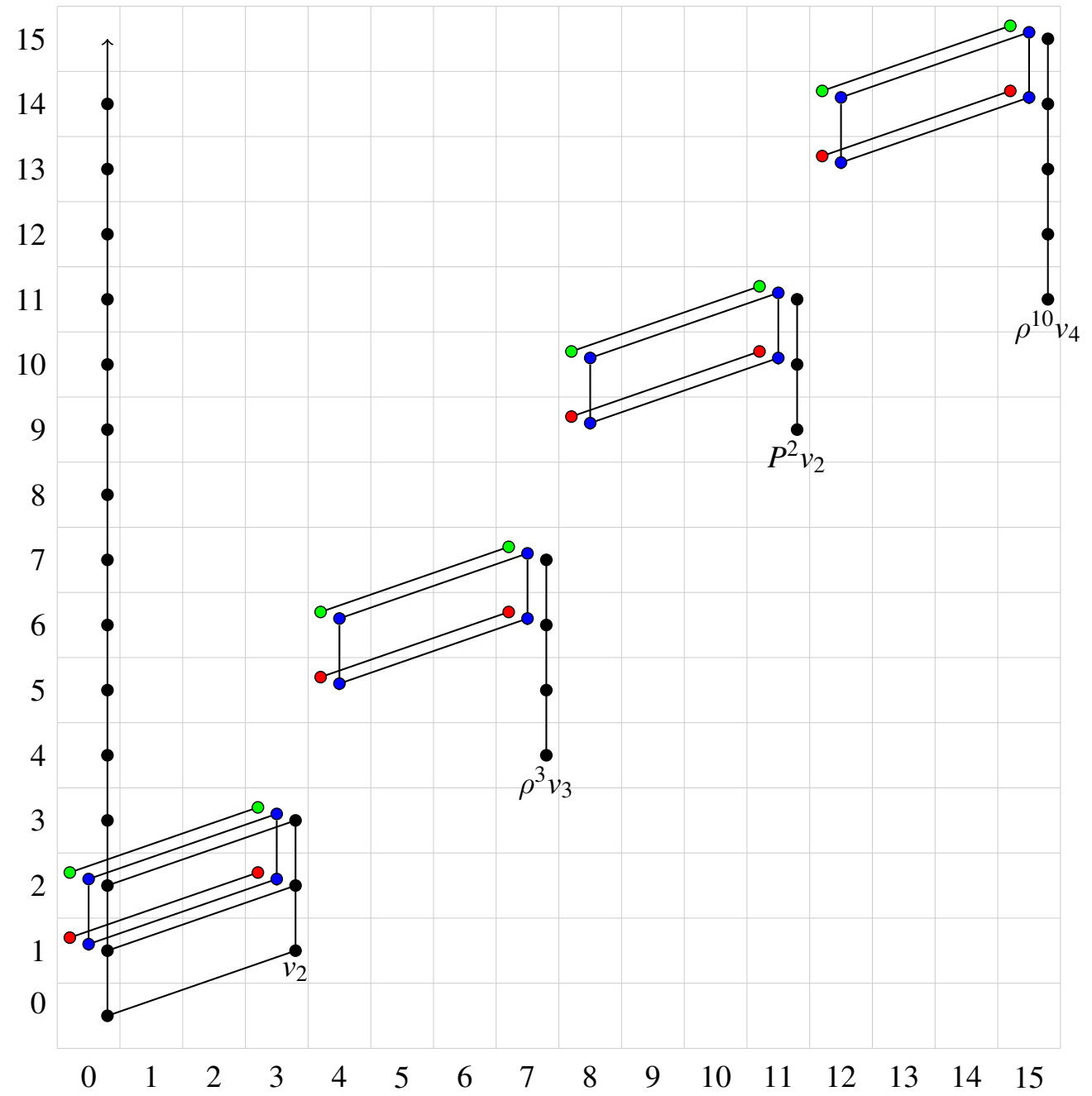

Figure 6: The $E_{\infty}$ page of $\mathfrak{M}(\mathbb{Q})\left[h_{1}^{-1}\right]$ up to Milnor-Witt stem 15 . The notational conventions of figure ?? apply here. 